\title{
NOTAS Y DISFUNCIONES EN LA DISTRIBUCION DEL PROFESORADO \\ UNIVERSITARIO EN ESPAÑA: \\ UNA APROXIMACION
}

Carlos Velasco Murviedro y María Pía Velázquez Gutiérrez

\section{Introducción}

Todos somos conscientes de los grandes males que aquejan a la Universidad española, y a quien no lo fuera le bastaría con hacer cualquier tipo de investigación o estudio sobre su estructura, organización y funcionamiento para darse cuenta de las disfunciones que existen en el sistema universitario. Uno de los más importantes, y que incide más directamente en la formación del alumno, es la distribución del profesorado por centros universitarios y áreas de estudio.

La distribución actual del profesorado universitario es muy deficiente; consecuencia de ello es que, frente a centros que cuentan con un número suficiente (e incluso excesivo) de profesores, existen otros cuya deficiencia es notoria, repercutiendo de una manera negativa en el aprovechamiento que de sus estudios hacen los alumnos y desaprovechándose los recursos, tanto humanos como económicos, disponibles. En un momento en que se habla tanto de la «rentabilidad» de nuestra Universidad y de su coste social, es necesario aprovechar bien todo lo que se dispone y obtener con ello el máximo rendimiento. 
El presente estudio tiene como finalidad mostrar esta desigual distribución del profesorado, poniendo en relación las horas lectivas que (según el número de profesores) se imparten en cada centro universitario, con las que son necesarias según el alumnado y el plan de estudios.

Sin embargo, las conclusiones a las que se llega deben ser necesariamente matizadas y servir como mera aproximación a la situación real del momento presente, pues se ha partido de una serie de limitaciones (metodológicas, temporales, de base de datos, etc.) que condicionan, lógicamente, los resultados. Por ejemplo, al haber transcurrido dos años (los datos son del curso 1981-82), no puede afirmarse que la situación actual sea la misma, y máxime con la reacomodación a que el profesorado se está sometiendo el curso pasado y el actual. A pesar de todo lo anterior, creemos que el análisis de los datos ofrecidos puede servir como un indicativo bastante aceptable de la compleja situación existente en cuanto a su distribución, como demostración palpable de la falta de adecuación entre los recursos existentes y el uso que se hace de ellos, y para mostrar la necesidad del cambio que debe operarse en la organización y estructura de nuestra Universidad para, de esta manera, hacerla más racional y operativa.

En este sentido, la recientemente aprobada Ley de Reforma Universitaria posibilita esas modificaciones que necesita el sistema universitario, y es de esperar que, a partir de su puesta en vigor y su desarrollo normativo, los cambios comiencen a producirse.

Las limitaciones a que nos referimos sirven también como muestra del desorden existente en el tema que nos ocupa; así, los planes de estudio carecen de la más mínima homogeneidad, pues las mismas carreras no tienen igual plan de estudios; éstos, en ciertos casos, no existen oficialmente (es decir, no están publicados en el $B O E$ ) y, en otros, no hacen ninguna distinción entre las horas de enseñanza teóricas y las prácticas, lo cual hace imposible un estudio fiable de horario real del alumnado; tampoco son homogéneas las áreas de estudios, habiendo algunas, como filosofía, psicología, etc., que mientras en una Universidad tienen rango de Facultad, en otras es sólo Sección o Especialidad, siendo, por tanto, muy arriesgado decir, de manera tajante, que tal o cual estudio está mal dotado de profesorado. Otro problema importante a la hora de realizar el trabajo fue el de la fiabilidad de los datos que sobre el número de profesores y alumnos se poseían, pues el profesorado en ocasiones no estaba desglosado por categorías; el alumnado, en ciertos casos, se ofrecía para toda la Facultad en su conjunto, sin distinciones entre las diferentes secciones, y los alumnos de doctorado (dada su compleja distribución en asignaturas y cursillos) no se han podido tomar en consideración; esto, unido al retraso de las estadísticas oficiales (en junio de 1983, las cifras definitivas más actuales eran las del curso 1981-82), hace imposible afirmar, de una manera tajante, que ésa sea la situación actual de la distri. bución del profesorado en nuestra Universidad, aunque sí que se le aproxima 
mucho. Por ello, y a pesar de las dificultades evidentes que el tema entraña, se ha tratado de hacer un análisis de la situación del profesorado en base a los datos disponibles, a fin de ofrecer una panorámica, lo más fidedigna posible, de la Universidad bajo este aspecto.

\section{Metodología}

Se han tomado en consideración los dos tipos de profesores universitarios existentes, clasificados por el tipo de enseñanza que imparten: Profesorado Con Responsabilidad Docente (PRD), encargado de las clases teóricas, y Profesorado Sin Responsabilidad Docente (SRD), que es el que (teóricamente al menos) imparte la enseñanza práctica (seminarios, laboratorios, etc.).

Las horas lectivas semanales (HLS) de estos profesores varían según la clase de dedicación que tengan: así, para el PRD, el horario semanal es de tres horas para la dedicación normal, seis horas para la plena y nueve para la exclusiva. En el caso de los Encargados de Curso se ha estimado la media del horario semanal de todos ellos que impartieron durante el curso 1981-82, que fue de siete horas; se ha trabajado con esta cifra, pues dadas las diferentes categorías existentes en este tipo de profesorado (desde tres horas a la semana hasta doce) y a que en la mayoría de los casos se disponía sólo del total de Encargados de Curso, sin especificar la dedicación que tenían, era casi imposible obtener datos fiables por cada colectivo.

En el Profesorado Sin Responsabilidad Docente (SRD) existen dos tipos de profesores: los Ayudantes, con un horario semanal de seis, doce y dieciocho horas, y los Maestros de Taller, cuyo horario es de veinticinco, treinta y cuarenta horas semanales (las tres cifras, en ambos casos, se corresponden con los tres tipos de dedicación).

Las cifras de alumnado con que se ha trabajado han sido las que nos ofrecían los datos de matrícula oficial, pues la libre está en franca regresión y, en la mayoría de los casos estudiados, su número no era apenas representativo. Como se dijo anteriormente, los alumnos de doctorado tampoco se han tomado en consideración.

El tamaño de los grupos que se han formado con los alumnos existentes ha sido éste (la primera cifra afecta a los cursos del primer ciclo, y la segun$\mathrm{da}$, al segundo ciclo):

\section{PRD}

Ciencias

Facultades y ETS $=60 / 30$ (la segunda cifra es para la especialización).

Escuelas universitarias (EE. UU.) $=50$ (todos los cursos igual) 
Letras

$$
\begin{aligned}
& \text { Facultades }=100 / 50 \\
& \text { Escuelas universitarias }=70
\end{aligned}
$$

SRD

\section{Ciencias}

Facultades y ETS $=20 / 15$

Escuelas universitarias $=25$

Letras

Aunque, lógicamente, es discutible la adopción de estas cifras (a pesar del tamaño reducido de los grupos, creemos que superado, en la mayor parte de los casos, en la realidad), que sirven para obtener el sobrante o déficit de las HLS a escala nacional, no influyen para casi nada en la situación relativa entre Universidades, de forma que si hay unas bien dotadas de profesorado y otras mal, con el tamaño de los grupos considerado, las diferencias se mantendrían (en porcentaje y en signo) al variar los módulos. Esto es importante que se tenga presente, sobre todo con vistas a las comparaciones y desigualdades existentes.

Se ha aplicado siempre en los cálculos el principio de "mayor cobertura» para las distintas especialidades, queriéndose indicar con él que, en caso de duda, se han favorecido las HLS necesarias para cubrir los cursos o especialidades. Como casos concretos podemos citar:

- El más mínimo exceso de un solo alumno sobre los 25, 30, 35, 50, etcétera, alumnos/grupo implica, necesariamente, la creación de un nuevo grupo teórico para atenderle y de las HLS para cubrirle.

- A partir de cuarto y quinto cursos (o de tercero, a veces), cuando las especialidades existentes (la mayor parte con bastantes asignaturas comunes) exceden del número de grupos teóricos que se deducen del número de alumnos, se ha trabajado con la primera cifra, a fin de cubrir el máximo teórico de grupos, y sin tener en cuenta las asignaturas repetidas.

- Aunque a veces es probable que en los cursos primero, segundo y tercero haya asignaturas comunes de facto, se ha supuesto la total independencia entre unas y otras Secciones a la hora de formar grupos mínimos de $\mathrm{X}$ alumnos/grupo.

- Cuando hay "curso de adaptación» para el acceso al segundo ciclo, se han añadido a los alumnos del tercer curso $\mathrm{y}$, después, se han hecho grupos con todos ellos. 
El déficit o superávit de HLS en cada centro universitario se ha obtenido en base a cálculos realizados para cada área de estudios, distinguiendo entre PRD y SRD.

El cuadro 1 explica el método utilizado. Para cada centro universitario se toma el total de alumnos por curso, desglosados por Secciones o Especialidades o en conjunto, según nos sean facilitados los datos (columna 1); se dividen por el tamaño del grupo que le corresponda (columna 2) y, en este caso, se utiliza la cifra "100" por ser Facultad, área de «Letras», primer ciclo y corresponder el estudio al Profesorado Con Responsabilidad Docente (visto ya más atrás). La primera cifra de la columna 3 es el resultado de la operación anterior aplicando el principio, ya visto, de «mayor cobertura»; ello quiere decir que, aunque el tamaño del grupo sea 100 , se ha creado otro nuevo con los 17 alumnos sobrantes; por otra parte, la segunda cifra de la referida columna corresponde al número de grupos que fija el plan de estudios del centro que se trate (en este caso, la Facultad de Ciencias de la Información de la Universidad Complutense de Madrid), pues habrá veces que esta cifra sea mayor que la inmediatamente anterior y habrá que tenerla en cuenta. En la columna siguiente (4) se reflejan las Horas Lectivas Semanales que para ese curso determina el plan de estudios (esta cifra se obtiene al sumar las HLS de todas las asignaturas que componen el curso), las cuales, al multiplicarse (columna 5) por la cifra mayor de las dos que aparecen en la columna 3 (el motivo de esta elección es el de dar un mayor margen de necesidades de profesorado, para así favorecer al Centro objeto del estudio), nos dará el total de HLS necesarias para el curso y la Sección o Especialidad que se trate. En el ejemplo que figura en el cuadro 1, habría que sumar las cifras correspondientes a las tres Secciones que tiene la facultad y, de esta manera, hallar que las HLS necesarias para el primer curso de Ciencias de la Información son 480. Realizando las mismas operaciones para los cuatro cursos restantes, y sumando sus resultados, obtendremos 1.580 HLS necesarias para el total de la Facultad (parte inferior del cuadro). Esta cifra es la que debemos restar de la que aparece en la parte superior derecha del ejemplo considerado, que son las HLS pagadas para esa Facultad. Al llegar a este punto es necesario hacer una clara distinción entre ambos conceptos, puesto que es fundamental para comprender el objeto de este estudio $y$, al mismo tiempo, extraer sus conclusiones.

Las HLS pagadas son las qus deben de impartir los profesores del Centro o, al menos, las que se les pagan con el fin de que se impartan. Para obtenerlas se multiplica el número de ellos (agrupándolos por categorías) por las horas lectivas semanales que fije su dedicación; es decir, y siguiendo con el ejemplo expuesto, en la Facultad de Ciencias de la Información hay dos catedráticos con dedicación normal $(2 \times 3)$, ocho con plena $(8 \times 6)$ y siete con exclusiva $(7 \times 9)$; haciendo lo mismo con las restantes categorías de 


\section{CUADRO 1}

Adecuación entre las boras lectivas semanales (HLS) existentes

(Necesarias y pagadas)

Universidad:

Facultad: Ciencias de la Información Sección:

(Curso 1981-82)
HLS pagadas al Profesorado Con Responsabilidad Docente $(\mathrm{PRD})=(2 \times 3)+$ $+(8 \times 6)+(7 \times 9)+(2 \times 3)+(6 \times 6)+(10 \times 9)+(30 \times 6)+(22 \times 9)+$ $+(93 \times 7)=6+48+63+6+36+90+180+198+651=1.278$.

$H L S$ necesarias para cubrir los grupos de $100 / 50$ alumnos.

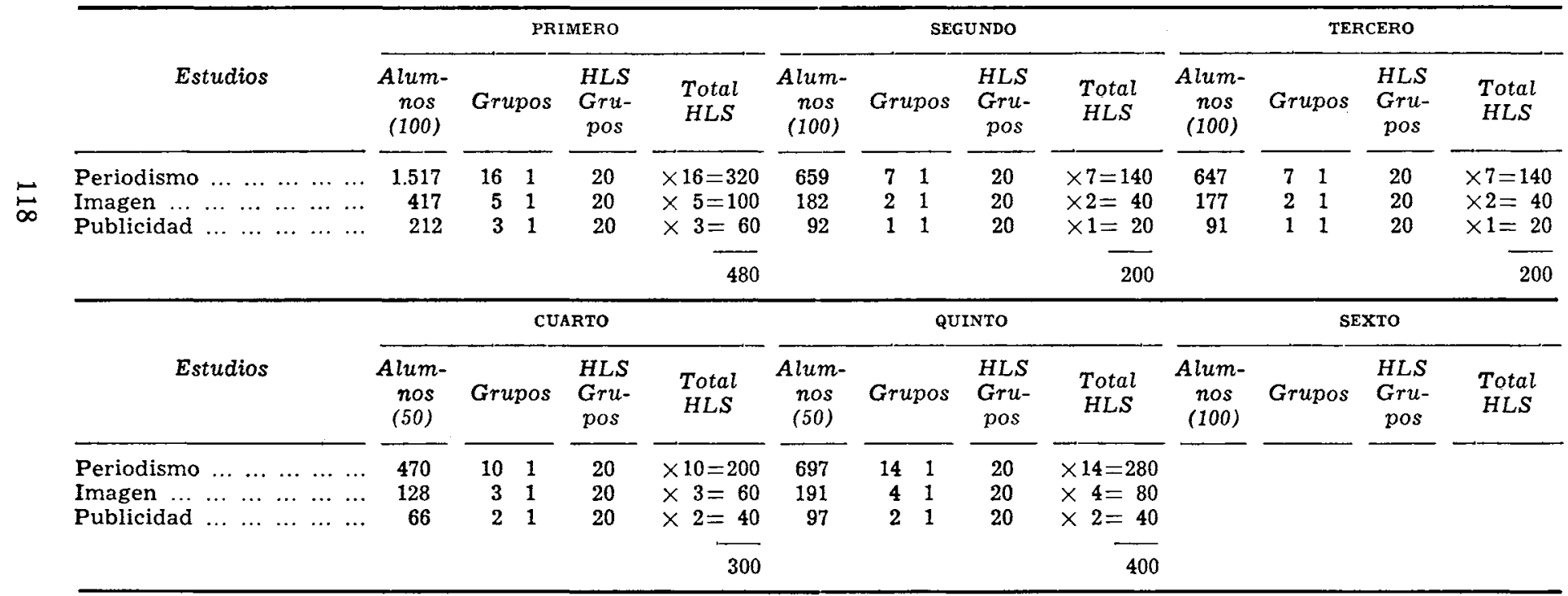

Total HLS necesarias $=480+200+200+300+400=1.580$.

Número de alumnos $=5.643$.

$1.278-1.580=-302$ HLS déficit $-19,11 \%$ déficit 
Profesorado Con Responsabilidad Docente, y sumando todas las cifras, nos encontramos con que para esa Facultad habrá 1.278 HLS presupuestadas, pagadas y susceptibles, pues, de ser empleadas para la docencia de los grupos de alumnos existentes.

Las HLS necesarias (ya hemos visto cómo se obtienen) son las que necesita la Facultad para cumplir el horario fijado en su Plan de Estudios, teniendo en cuenta, al mismo tiempo, el número de alumnos que cursan sus estudios en dicho Centro. Del resultado de confrontar ambas cifras (HLS pagadas y HLS necesarias) depende el si ese centro tiene superávit o déficit, es decir, si realmente el profesorado adscrito a esa área de estudio es suficiente (cantidad de HLS pagadas superior a las necesarias) o si, por el contrario, serían necesarios más profesores para atender adecuadamente al alumnado y cumplir el Plan de Estudios. La situación óptima y que reflejaría una buena distribución del profesorado, sería (suponiendo una correcta definición de los módulos alumnos/profesor) la de que las dos cifras fueran iguales, ya que de esta manera a una demanda de determinadas HLS necesarias se ofrecería la misma cantidad de HLS pagadas. Pretendemos no ser utópicos ni creer que puede darse total y realmente esta situación, pero sí debemos intentar que llegue a ser posible, disminuyendo al máximo las diferencias existentes: es por ello que creemos que la información que se ofrece a lo largo de este trabajo puede ser de utilidad a la hora de llevar a cabo una redistribución del profesorado, orientando la masa sobrante desde los estudios con superávit hacia aquellos otros con claro déficit.

\section{Facultades}

Dentro del trofesorado con responsabilidad docente (PRD), la nota general más destacable es que, a nivel nacional, faltan horas lectivas semanales por un monto de 12.946 y un porcentaje de $-11,69$ por 100 (cuadro 2). Por Universidades, son pocas las que disponen de sobrantes, aunque algunas resultan significativas: Alcalá de Henares $(59,15$ por 100$)$, Santiago $(23,18)$, Extremadura (21,48), Barcelona Autónoma (16,57), Málaga $(15,12)$, Santander $(9,01)$, Murcia $(6,73)$, Baleares $(6,18)$, La Laguna $(5,89)$ y Madrid Autónoma $(0,92)$.

En cuanto a las Universidades con mayores déficits de HLS, son éstas: Valencia $(-38,95$ por 100$)$, Zaragoza $(-36,99)$, Granada $(-31,75)$, Cádiz $(-30,81)$, Córdoba $(-26,81)$, Sevilla $(-19,58)$, Valladolid $(-17,87)$, Alicante $(-14,5)$, León $(-14,27)$, Barcelona Central $(-13,61)$, Oviedo $(-12,76)$, País Vasco $(-7,37)$, Madrid Complutense $(-6,47)$ y Salamanca $(-1,38)$.

Las anteriores consideraciones se modifican algo (bastante poco) al alza 


\section{CUADRO 2}

Facultades

Curso 1981-82
Resumen de las HLS de Facultades, según Universidades

Profesorado con responsabilidad docente (PRD)

\begin{tabular}{|c|c|c|c|c|c|c|c|c|c|}
\hline \multirow[t]{2}{*}{ Universidades } & \multirow[b]{2}{*}{ Alumnos } & \multirow[b]{2}{*}{$\begin{array}{l}\text { N. pro- } \\
\text { fesores }\end{array}$} & \multirow[b]{2}{*}{$\begin{array}{l}\text { N. espe- } \\
\text { cialid. }\end{array}$} & \multirow[b]{2}{*}{$\begin{array}{c}\text { HLS } \\
\text { pagadas }\end{array}$} & \multirow[b]{2}{*}{$\begin{array}{c}H L S \\
\text { necesarias }\end{array}$} & \multicolumn{2}{|c|}{ DIFERENCIA } & \multicolumn{2}{|c|}{ EXCESOS POSITIVOS } \\
\hline & & & & & & $\begin{array}{c}\text { Valor } \\
\text { absolute }\end{array}$ & $\%$ & $\begin{array}{c}\text { Valor } \\
\text { absoluto }\end{array}$ & $\%$ \\
\hline Alcalá de Henares . ... ... & 3.279 & 240 & 8 & 1.808 & 1.136 & 672 & 59,15 & 672 & 59,15 \\
\hline 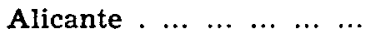 & 3.656 & 184 & 7 & 1.497 & 1.751 & -254 & $-14,5$ & 130 & 7,42 \\
\hline Baleares.$\ldots \ldots \ldots \ldots$ & 2.694 & 135 & 7 & 1.082 & 1.019 & 63 & 6,18 & 257 & 25,22 \\
\hline Barcelona Autónoma .. ... & 15.261 & 585 & 23 & 5.944 & 5.099 & 845 & 16,57 & 868,5 & 17,03 \\
\hline Barcelona Central $\ldots \ldots$ & 46.941 & 1.563 & 78 & 11.662 & 13.500 & -1.838 & $-13,61$ & 1.135 & 8,40 \\
\hline 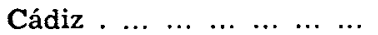 & 3.344 & 123 & 3 & 887 & 1.282 & -395 & $-30,81$ & 89 & 6,94 \\
\hline Córdoba $\ldots \ldots \ldots \ldots \ldots$ & 5.329 & 250 & 18 & 2.025 & 2.767 & -742 & $-26,81$ & 109 & 3,93 \\
\hline $\begin{array}{llllll}\text { Extremadura } & \ldots & \ldots & \ldots & \ldots\end{array}$ & 3.475 & 217 & 15 & 1.783 & 1.400 & 383 & 21,48 & 445 & 31,78 \\
\hline $\begin{array}{cccccc}\text { Granada } & \ldots & \ldots & \ldots & \ldots & \ldots\end{array}$ & 19.051 & 598 & 31 & 4.347 & $6.369,5$ & $-2.022,5$ & $-31,75$ & 201 & 0,31 \\
\hline La Laguna $\ldots \ldots \ldots \ldots$ & 8.938 & 508 & 37 & 4.085 & $3.857,5$ & 227,5 & 5,89 & 1.228 & 31,83 \\
\hline 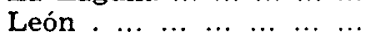 & 2.946 & 105 & 5 & 883 & 1.030 & -147 & $-14,27$ & 173 & 16,79 \\
\hline Madrid Autónoma $\ldots . .$. & 20.619 & 851 & 43 & 6.225 & 6.168 & 57 & 0,92 & 1.582 & 25,64 \\
\hline Madrid Complutense .. ... & 57.606 & 2.457 & 102 & 17.374 & $18.554,75$ & $-1.180,75$ & $-6,47$ & $2.340,75$ & 12,61 \\
\hline 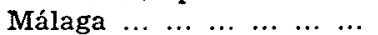 & 8.472 & 340 & 21 & 2.710 & 2.354 & 356 & 15,12 & 874,5 & 37,14 \\
\hline $\begin{array}{llllll}\text { Murcia } & \ldots & \ldots & \ldots & \ldots & \ldots\end{array}$ & 8.576 & 367 & 28 & 2.891 & $2.708,5$ & 182,5 & 6,73 & 569 & 21 \\
\hline $\begin{array}{llllllll}\text { Oviedo } & \ldots & \ldots & \ldots & \ldots & \ldots & \ldots\end{array}$ & 8.435 & 335 & 24 & 2.723 & $3.121,5$ & $-398,5$ & $-12,76$ & 441,5 & 14,14 \\
\hline $\begin{array}{llllll} & \text { País Vasco } & \ldots & \ldots & \ldots & \ldots\end{array}$ & 13.289 & 718 & 37 & 5.368 & $5.795,5$ & $-427,5$ & $-7,37$ & 313 & 540 \\
\hline $\begin{array}{ccccc}\text { Salamanca } & \ldots & \ldots & \ldots & \ldots\end{array}$ & 11.171 & 469 & 23 & 3.850 & 3.904 & -54 & $-1,38$ & 482 & 12,34 \\
\hline $\begin{array}{lllllll}\text { Santander } & \ldots & \ldots & \ldots & \ldots & \ldots & \ldots\end{array}$ & 2.064 & 139 & 9 & 1.065 & 977 & 88 & 9,01 & 216 & 22,10 \\
\hline $\begin{array}{llllll}\text { Santiago } & \ldots & \ldots & \ldots & \ldots & \ldots\end{array}$ & 18.010 & 460 & 39 & 2.697 & 3.511 & -814 & 23,18 & 257 & 7,31 \\
\hline 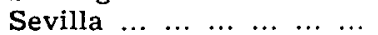 & 17.460 & 577 & 34 & 4.533 & 5.637 & -1.104 & $-19,58$ & 520 & 9,22 \\
\hline $\begin{array}{cccccc}\text { Valencia } & \ldots & \ldots & \ldots & \ldots & \ldots\end{array}$ & 27.360 & 710 & 53 & 5.560 & 9.108 & -3.548 & $-38,95$ & 457 & 5,02 \\
\hline Valladolid $\ldots \ldots \ldots \ldots$ & 10.268 & 384 & 29 & 3.034 & $3.694,25$ & $-660,25$ & $-17,87$ & 25 & 0,67 \\
\hline $\begin{array}{llllll}\text { Zaragoza } & \ldots & \ldots & \ldots & \ldots & \ldots\end{array}$ & 17.164 & 458 & 25 & 3.806 & 6.041 & -2.235 & $-36,99$ & 402 & 6,65 \\
\hline TOtaL $\ldots \ldots \ldots \ldots \ldots$ & 335.768 & 12.773 & 699 & 97.839 & $110.785,5$ & -12.946 .5 & $-11,69$ & $13.787,25$ & 12,44 \\
\hline
\end{tabular}


si se eliminan los déficits de los estudios en que aparezcan y se tienen en cuenta solamente los sobrantes de HLS. El porqué de este enfoque (dos últimas columnas del cuadro 2) está en el hecho de que sumar algebraicamente valores positivos y negativos de las HLS de las Universidades da una visión de conjunto, aunque no corresponde totalmente a la situación real, toda vez que en aquellos casos en que se paguen más HLS de las necesarias podrán congelarse los recursos dedicados al profesorado, pero ha sido, hasta el momento, inviable pensar en que parte de estos sobrantes sirvieran para trasladarse y cubrir los déficits de las Universidades y estudios con escasez de profesorado. Parece, pues, más acorde con la realidad actual, a la hora de analizar la distribución docente, observar este sobrante de valores absolutos, pues indica que, además de existir superávits generalizados y desiguales, no han podido aplicarse para «amortizar» las carencias existentes, que han pedido (bastante justificadamente) la cobertura de sus deficiencias ex novo y no a cargo del resto de Universidades.

En base a estos criterios, lo que se observa es un superávit de 12,44 por 100 de HLS, que significa, en valores absolutos, 13.787 HLS sobrantes. Por Universidades, las mejores situadas son, con altibajos, las mismas que se han visto más atrás: Alcalá de Henares $(59,15$ por 100$)$, Málaga $(37,14)$, La Laguna (31,83), Extremadura (31,78), Madrid Autónoma $(25,64)$, Baleares $(25,22)$, Santander $(22,10)$, Murcia $(21,00)$, etc.; en cuanto a aquellas a las que, aun sobrándoles HLS, lo son en pequeño porcentaje, tenemos a Granada $(0,67$ por 100), Valladolid $(0,67)$, Córdoba $(3,93)$, Valencia $(5,02)$, País Vasco $(5,40)$, Zaragoza $(6,65)$, Cádiz $(6,94)$ y Alicante $(7,42)$.

La comparación entre estas columnas y las dos inmediatamente anteriores puede ser una muestra de lo que realmente significa el déficit primero de HLS $(-11,69)$ en relación con el sobrante segundo $(12,44)$ a escala nacional. Por lo que parece, en las Facultades españolas faltan HLS del PRD; pero también es cierto que las HLS existentes están muy mal distribuidas, no sólo entre diferentes Universidades (primer y segundo párrafos de este apartado), sino, dentro de ellas, entre sus diferentes estudios, que es lo que hace que todas las Universidades españolas sin distinción (dos últimas columnas del cuadro 2) tengan sobrantes de HLS en algunos de sus estudios, que agravan y perjudican su comparación con aquellos deficitarios. Los casos de Alicante, León, Oviedo y Sevilla serían, a este respecto, los más llamativos, pues pasan de tener unos importantes déficits «globales» y algebraicos en HLS $(-14,5$, $-14,27,-12,76$ y $-19,58)$ a unos superávits discretos y de importancia en las dos últimas columnas $(7,42,16,79,14,14$ y 9,22); dicho en otras palabras: el cambio de signo de estas Universidades nos está indicando que, a pesar de su global situación deficitaria, tienen unos estudios dotados muy desigualmente, de forma que mientras a unos les sobran, a otros les faltan.

De estos estudios en cada Universidad es precisamente de lo que se va 


\section{CUADRO 3}

Facultades

Resumen de las HLS de Facultades, según Universidades

Curso 1981-82

Profesorado sin responsabilidad docente (SRD)

\begin{tabular}{|c|c|c|c|c|c|c|c|c|c|}
\hline \multirow[t]{2}{*}{ Universidades } & \multirow[b]{2}{*}{ Alumnos } & \multirow[b]{2}{*}{$\begin{array}{r}\text { N." pro- } \\
\text { fesores }\end{array}$} & \multirow[b]{2}{*}{$\begin{array}{l}\text { N.o espe- } \\
\text { cialid. }\end{array}$} & \multirow[b]{2}{*}{$\begin{array}{c}H L S \\
\text { pagadas }\end{array}$} & \multirow[b]{2}{*}{$\begin{array}{c}H L S \\
\text { necesarias }\end{array}$} & \multicolumn{2}{|c|}{ DIFERENCIA } & \multicolumn{2}{|c|}{ EXcesos POSITIVOS } \\
\hline & & & & & & $\begin{array}{c}\text { Valor } \\
\text { absoluto }\end{array}$ & $\%$ & $\begin{array}{c}\text { Valar } \\
\text { absoluto }\end{array}$ & \% \\
\hline Alcalá de Henares . ... ... & 3.279 & 61 & 8 & 1.056 & $\mathbf{5 7 2 , 5}$ & 483,5 & 84,45 & 483,5 & 84,45 \\
\hline Alicante.$\ldots \ldots \ldots \ldots$ & 3.656 & 53 & 7 & 366 & 776 & -410 & $-52,83$ & - & - \\
\hline Baleares.$\ldots \ldots \ldots \ldots$ & 2.694 & 35 & 7 & 360 & 656 & -296 & $-45,12$ & - & - \\
\hline Barcelona Autónoma .. ... & 15.261 & 452 & 23 & 4.086 & $3.405,97$ & 680,03 & 19,96 & 680,03 & 19,96 \\
\hline Barcelona Central . ... ... & 46.941 & 818 & 76 & 7.106 & $4.857,5$ & $2.248,5$ & 46,28 & $2.248,5$ & 46,28 \\
\hline Cádiz.$\ldots \ldots \ldots \ldots$ & 3.344 & 69 & 3 & 276 & 175 & 101 & 57,71 & 101 & 57,71 \\
\hline 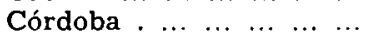 & 5.329 & 148 & 18 & 1.170 & 682 & 488 & 71,55 & 506 & 74,19 \\
\hline Extremadura $\ldots \ldots \ldots$ & 3.475 & 94 & 15 & 438 & 470,5 & $-32,5$ & $-6,90$ & - & - \\
\hline Granada $\ldots \ldots \ldots \ldots \ldots$ & 19.051 & 561 & 30 & 6.598 & 3.474 & 3.124 & 89,93 & 3.124 & 89,93 \\
\hline La Laguna $\ldots \ldots \ldots$ & 8.938 & 221 & 34 & 2.838 & 2.559 & 279 & 10,90 & 856 & 39,21 \\
\hline 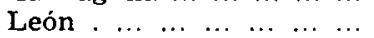 & 2.946 & 74 & 7 & 636 & 406 & 230 & 56,65 & 230 & 56,65 \\
\hline Madrid Autónoma . ... ... & 20.619 & 276 & 43 & 4.528 & 4.988 & -460 & $-9,22$ & 1.771 & 45,50 \\
\hline Madrid Complutense . ... & 57.606 & 1.530 & 102 & 4.581 & $6.568,5$ & $-1.987,5$ & $-30,25$ & 730,5 & 11,12 \\
\hline 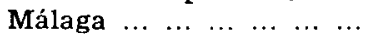 & 8.472 & 195 & 21 & 2.064 & 872 & 1.192 & 136,69 & 1.254 & 143,80 \\
\hline $\begin{array}{lllllll}\text { Murcia } & \ldots & \ldots & \ldots & \ldots & \ldots & \ldots\end{array}$ & 8.576 & 174 & 27 & 1.488 & 1.413 & 75 & 5,30 & 224 & 15,85 \\
\hline $\begin{array}{lllllll}\text { Oviedo } & \ldots & \ldots & \ldots & \ldots & \ldots & \ldots\end{array}$ & 8.435 & 360 & 24 & 2.508 & 1.093 & 1.415 & 129,46 & 1.415 & 129,46 \\
\hline País Vasco $\ldots \ldots \ldots \ldots$ & 13.289 & 132 & 32 & 1.032 & 1.936 & -904 & $-46,69$ & 232 & 11,98 \\
\hline $\begin{array}{llllll}\text { Salamanca } & \ldots & \ldots & \ldots & \ldots & \ldots\end{array}$ & 11.171 & 438 & 23 & 1.490 & 1.207 & 283 & 23,44 & 292 & 24,19 \\
\hline 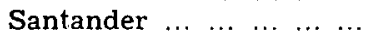 & 2.064 & 92 & 13 & 702 & 171 & 531 & 310,52 & 531 & 310,52 \\
\hline 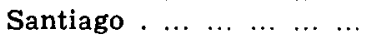 & 18.010 & 277 & 35 & 2.699 & 5.725 & -3.026 & $-52,85$ & 142 & 2,48 \\
\hline $\begin{array}{lllllll}\text { Sevilla } & \ldots & \ldots & \ldots & \ldots & \ldots & \ldots\end{array}$ & 17.460 & 463 & 34 & 5.263 & 3.622 & 1.641 & $\mathbf{4 5}, 30$ & 1.927 & 53,20 \\
\hline $\begin{array}{llllll}\text { Valencia } . & \ldots & \ldots & \ldots & \ldots & \ldots\end{array}$ & 27.360 & 463 & 50 & 4.020 & 4.370 & -350 & -80 & 452 & 10,34 \\
\hline $\begin{array}{llllll}\text { Valladolid } & \ldots & \ldots & \ldots & \ldots & \ldots\end{array}$ & 10.268 & 220 & 29 & 4.391 & $7.297,45$ & $-2.906,45$ & $-39,82$ & 18,25 & 0,25 \\
\hline $\begin{array}{llllll}\text { Zaragoza } & \ldots & \ldots & \ldots & \ldots & \ldots\end{array}$ & 17.164 & 499 & 25 & 1.866 & $1.690,2$ & 175,8 & 10,40 & 175,8 & 10,48 \\
\hline Total $\ldots \ldots \ldots$ & 335.768 & 7.705 & 686 & 61.562 & $58.987,62$ & $2.574,38$ & 4,36 & $17.393,56$ & 29,48 \\
\hline
\end{tabular}


a hablar a continuación, y de su grado de homogeneidad de HLS dentro de cada distrito, aunque no se acompañan los datos concretos*. Esta podría ser una clasificación de ellas según sus estudios:

- Sobrantes generalizados, aunque desiguales: Alcalá de Henares, Barcelona Autónoma y Murcia.

- Déficits generalizados y similares: Alicante, Granada y Valencia.

- Desiguales cifras y signo: Baleares, Barcelona Central, Cádiz, Córdoba, Extremadura, La Laguna, León, Madrid Autónoma, Madrid Complutense, Málaga, Oviedo, País Vasco, Salamanca, Santander, Santiago, Sevilla, Valladolid y Zaragoza.

Como resumen de esto, puede decirse que los déficits de HLS son muy generales y que salpican casi todas las Universidades, aunque algunas se salvan por sus cantidades sobrantes de HLS. Pasando ya al análisis concreto de la estructura de estas HLS según los diferentes estudios, el resumen de las principales conclusiones se recoge en el cuadro 4, en su segunda columna.

En él pueden observarse dos grupos claramente delimitados, que hacen referencia al signo del porcentaje de variación de HLS. En primer lugar se encuentran los estudios superavitarios: Políticas y Sociología $(108,37$ por 100 de HLS sobrantes y 1 lugar donde puede estudiarse), Geológicas (38,68 y 3), Físicas (37,36 y 5), Ciencias (36,38 y 13), Psicología $(31,86)$, Matemáticas (24,50 y 6), Bellas Artes (23,96 y 6), Química (20,62 y 12), Geografía e Historia $(15,15$ y 7$)$, Filosofía y Letras $(3,68$ y 16) y Filosofía y Ciencias de la Educación $(3,10$ y 7$)$.

En cuanto a los estudios con situación negativa, son menos que los del grupo anterior: Farmacia ( $-41,69$ por 100 de déficit de HLS y 9 posibles lugares de estudio), Económicas y Empresariales (-37,67 y 15), Medicina $(-31,86$ y 22$)$, Derecho $(-25,84$ y 23$)$, Biológicas $(-22,04$ y 9$)$, Ciencias de la Información $(-15,08$ y 2$)$ y Filología $(-1,87$ y 7$)$.

A la vista de esta información, ¿cómo puede aplicarse la contradicción entre número abundante de estudios con sobresalientes de HLS (más que los negativos), y un volumen global de HLS negativo para todas las Universidades (el $-11,69$ por 100 ya visto más atrás)? La única razón de ello estriba en que aquellos estudios con déficits de HLS vistos en último lugar y que resulten menos numerosos que los superavitarios son, por así decir, «muy negativos"; es decir, no sólo tienen unas HLS necesarias mayores que las pagadas, sino que la cuantía es bastante importante, de forma que el total de estas deficiencias de HLS de todos los estudios compensa y supera la aparente

* Ello se debe a la enorme extensión de los cuadros que contienen dicha información y que, para los interesados, se encuentran disponibles en la Dirección General de Enseñanza Universitaria. 
bonanza de los numerosos estudios con sobrantes de HLS, y hace que aquéllos se hallen deficitarios no sólo en términos relativos, sino también absolutos.

\section{CUADRO 4}

Resumen de las HLS de Facultades, según estudios

Curso $1981-82$

\begin{tabular}{|c|c|c|c|}
\hline \multirow{2}{*}{ Estudios. Facultades } & & PRD & SRD \\
\hline & $\begin{array}{l}\text { N. de lu- } \\
\text { gares don- } \\
\text { de se es- } \\
\text { tudia }\end{array}$ & $\begin{array}{l}\text { Porcentaje de } \\
\text { variación } \\
\text { de HLS }\end{array}$ & $\begin{array}{l}\text { Porcentaje de } \\
\text { variación } \\
\text { de HLS }\end{array}$ \\
\hline$\ldots \ldots c c c$ & 13 & 36,38 & 18,25 \\
\hline 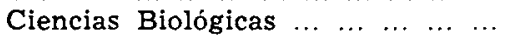 & 9 & $-22,04$ & $-17,78$ \\
\hline $\begin{array}{lllllll}\text { Ciencias Físicas } & \ldots & \ldots & \ldots & \ldots & \ldots & \ldots\end{array}$ & 5 & 37,36 & 149,75 \\
\hline 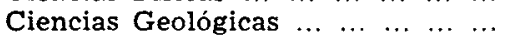 & 3 & 38,68 & 131,82 \\
\hline 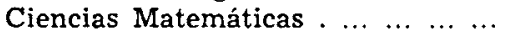 & 6 & 24,50 & 61,76 \\
\hline 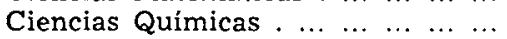 & 12 & 20,62 & 25,68 \\
\hline Ciencias Económicas y Empresa- & & & \\
\hline $\begin{array}{llllllllll}\text { riales } & \ldots & \ldots & \ldots & \ldots & \ldots & \ldots & \ldots & \ldots & \ldots\end{array}$ & 15 & $-37,67$ & $-44,08$ \\
\hline $\begin{array}{lllllllll}\text { Medicina } & \ldots & \ldots & \ldots & \ldots & \ldots & \ldots & \ldots & \ldots\end{array}$ & 22 & $-31,86$ & $-5,11$ \\
\hline $\begin{array}{llllllllll}\text { Derecho } & \ldots & \ldots & \ldots & \ldots & \ldots & \ldots & \ldots & \ldots & \ldots\end{array}$ & 23 & $-25,84$ & $-29,52$ \\
\hline $\begin{array}{cccccccc}\text { Farmacia } & \ldots & \ldots & \ldots & \ldots & \ldots & \ldots & \ldots\end{array}$ & 9 & $-41,69$ & $-14,29$ \\
\hline Filosofía y Letras $\ldots \ldots \ldots \ldots$ & 16 & 3,68 & $\mathbf{5 7 , 1 4}$ \\
\hline 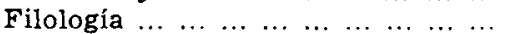 & 7 & $-1,87$ & 65,96 \\
\hline Filosofía y $\mathrm{C}$. de la Educación ... & 7 & 3,10 & $-15,08$ \\
\hline Ciencias de la Información . ... ... & 2 & $-15,08$ & $\mathrm{~N} / \mathrm{C}$ \\
\hline $\begin{array}{lllllllll}\text { Psicología } & \ldots & \ldots & \ldots & \ldots & \ldots & \ldots & \ldots & \ldots\end{array}$ & 1 & 31,86 & $\mathrm{~N} / \mathrm{C}$ \\
\hline Políticas y Sociología .... ... ... ... & 1 & 108,37 & $\mathrm{~N} / \mathrm{C}$ \\
\hline Geografia e Historia $\ldots \ldots \ldots \ldots \ldots$ & 7 & 15,15 & 537,09 \\
\hline $\begin{array}{llllllll}\text { Bellas Artes } & \ldots & \ldots & \ldots & \ldots & \ldots & \ldots & \ldots\end{array}$ & 6 & 23,96 & $-0,84$ \\
\hline
\end{tabular}

Pasando ahora al segundo tipo de elementos docentes, el profesorado sin responsabilidad docente ( $S R D$ ), la primera cifra a destacar (cuadro 3 ) es la referida a la suma algebraica de HLS, que es del 4,36 por 100 de HLS sobrantes a escala nacional para este tipo de profesorado, y que significan, en valores absolutos, 2.574 HLS (columnas 6 y 7). Por Universidades, existen dos grupos, según el signo; las principales son de carácter positivo, y entre ellas destacan: Santander (310,52 por 100), Málaga $(136,69)$, Oviedo $(129,46)$, Granada $(89,93)$, Alcalá de Henares $(84,45)$, Córdoba $(71,55)$, Cádiz $(57,71)$ y León $(56,65)$.

Por lo que se refiere a los déficits, éstas son las Universidades más notables: Valencia $(-80$ por 100$)$, Santiago $(-52,85)$, Alicante $(-52,83)$, País Vasco $(-46,69)$, Baleares $(-45,12)$, Valladolid $(-39,82)$, Madrid Complutense $(-30,25)$ y Madrid Autónoma $(-9,22)$.

Si nos centramos ahora en las dos últimas columnas de este mismo cuadro, se obtienen unas cifras de sólo los valores absolutos (ya explicados al hablar 
del PRD). Según ellas, si se hace abstracción de los déficits de HLS de los diferentes estudios que los tengan en las distintas Universidades, y sólo se cuentan los sobrantes existentes, el valor del anterior superávit nacional de HLS se ve notablemente incrementado, hasta un porcentaje del 29,48 por 100 y unos valores absolutos de 17.393 HLS, lo cual indica que los casos de sobrantes y los de déficits están también mal distribuidos, al no poder aplicarse, hasta ahora, aquéllos para amortizar éstos y resultar más excedentes que los obtenidos de la suma algebraica.

Analizada esta información por Universidades, los datos son muy similares a la enumeración antes hecha con la suma algebraica nacional $(4,36$ por 100 de superávit de HLS), pues aunque existen unas pequeñas diferencias positivas para la mayor parte de las Universidades, no influyen para nada en la clasificación de ellas según el mayor o menor volumen de HLS sobrantes y de su porcentaje relativo.

Si ahora nos referimos a la naturaleza de los estudios en cada Universidad (homogeneidad o no de sus signos y cuantías), se pueden apuntar ciertos grupos (tampoco se acompañan datos concretos en este caso):

- Sobrantes generalizados y de cantidad: Alcalá de Henares, Autónoma de Barcelona, Central de Barcelona, Granada, Oviedo, Salamanca y Santander.

- Déficits generalizados y de entidad: Alicante, Baleares, Valencia y Valladolid.

- Sobrantes y déficits: Córdoba, La Laguna, Autónoma de Madrid, Santiago y Sevilla.

- Casos irregulares: Cádiz, Extremadura, León, Madrid Complutense, Málaga, Murcia, País Vasco y Zaragoza.

De lo anterior puede observarse una cierta generalización de las Universidades con estudios mayormente superavitarios en HLS de su profesorado $S R D$, distribuyéndose el resto entre algunos ejemplos deficitarios, los que mezclan ambos casos y los irregulares de comportamiento. En cuanto a los estudios propiamente dichos, se han resumido en la última columna del ya visto cuadro 4.

Por un lado, estarían los estudios claramente superavitarios que, en su conjunto, disponen de muchas más HLS pagadas que las necesarias: Geografía e Historia (; 537,09 por 100 de HLS sobrantes, con 7 lugares donde estudiarse!), Físicas (149,75 y 5), Geológicas (131,82 y 3), Filología (65,96 y 7), Matemáticas (61,76 y 6), Filosofía y Letras $(57,14$ y 16), Químicas $(25,68$ y 12) y Ciencias $(18,25$ y 13$)$.

Aparte de los «no consta» (N/C) de Ciencias de la Información, Psicología y Políticas y Sociología, el resto de los estudios se hallan con déficits de HLS: Económicas y Empresariales ( $-44,08$ por 100 de déficit y 15 lugares donde pueden cursarse), Derecho (-29,52 y 23 ), Biológicas ( $-17,78$ y 9 ), 
Filosofía y Ciencias de la Educación (-15,08 y 7), Farmacia (-14,29 y 9), Medicina $(-5,11$ y 22) y Bellas Artes $(-0,84$ y 6$)$.

Tal vez, como resumen de lo anterior, y agrupándolo con datos referidos al profesorado $P R D$, se podría decir que hay ciertas Facultades o estudios en nuestro país que, por término medio y al margen de las diferencias entre los diferentes distritos en que pueden cursarse, se hallan con notables sobrantes de HLS, tanto en el profesorado con responsabilidad docente (PRD) como en el que no tiene dicha responsabilidad (SRD). Estos estudios serían todas las ramas de Ciencias (salvo Biológicas), Geografía e Historia, Políticas y Sociología, Filosofía y Letras y Psicología.

A su lado, hay otro grupo de estudios que coinciden bastante exactamente en sus déficits globales de HLS del PRD y del $S R D$, y son: Económicas y Empresariales, Derecho, Farmacia, Biológicas y Medicina.

\section{Escuelas Técnicas Superiores (ETS)}

La nota más general a destacar dentro de este tipo de centros es el sobrante generalizado de horas lectivas semanales (HLS) en el profesorado con responsabilidad docente ( $\mathrm{PRD}$ ) en prácticamente todas las Universidades (véase cuadro 5). Solamente el País Vasco $(-3,9$ por 100), UNED $(-7,86)$, Santiago $(-13,60)$, Sevilla $(-18,98)$ y Autónoma de Barcelona $(-27,8)$ presentan unas HLS necesarias mayores que las pagadas al profesorado y, en cualquier caso, el volumen global (la suma algebraica) de los sobrantes y/o déficits es netamente favorable a aquéllos, en un porcentaje del 37,62 por 100 sobre las HLS necesarias.

Por Universidades, las mejores situadas en este panorama son: Oviedo (con un sobrante de 168,42 por 100 de HLS), Santander (123,60), Córdoba $(98,3)$, Valencia Politécnica $(52,48)$, Madrid Politécnica $(42,82)$ y Las Palmas Politécnica $(41,19)$. A pesar de estas cifras, conviene examinar una por una cada Universidad, pues estos porcentajes son, a su vez, el resultado de agrupar los sobrantes y/o los déficits de los diferentes estudios cursados en ellas, muchas veces en cantidades y signo muy desiguales.

En cuanto a las dos últimas columnas referidas a sólo los incrementos positivos de los diferentes estudios y Universidades, puede observarse cómo las mejor y peor situadas desde esta perspectiva coinciden casi totalmente con las enumeradas más atrás, con sólo pequeñas diferencias de porcentajes.

En el caso que nos ocupa, y salvo aquellos distritos universitarios con un solo estudio, como Autónoma de Barcelona, Córdoba, País Vasco, Santander, Sevilla, Zaragoza y UNED, el resto de las Universidades son bastante semejantes en la estructura de HLS de sus diferentes estudios, aunque coexistiendo dentro de ellas algunos con déficits y otros con superávit, y/o unos con valores muy altos con otros cercanos al cero (Oviedo, Santiago y Valladolid); en este sentido, las más homogéneas tal vez sean las Politécnicas de Valencia, Madrid, Las Palmas y, en menor medida, Barcelona y Oviedo. 


\section{CUADRO 5}

ETS

Curso 1981-82
Resumen de las HLS de las ETS, según Universidades

Profesorado con responsabilidad docente (PRD)

\begin{tabular}{|c|c|c|c|c|c|c|c|c|c|}
\hline \multirow[t]{2}{*}{ Universidades } & \multirow[b]{2}{*}{ Alumnos } & \multirow[b]{2}{*}{$\begin{array}{l}\text { N. pro- } \\
\text { fesores }\end{array}$} & \multirow[b]{2}{*}{$\begin{array}{l}N . e s- \\
\text { pecialid. }\end{array}$} & \multirow[b]{2}{*}{$\begin{array}{c}\text { HLS } \\
\text { pagadas }\end{array}$} & \multirow[b]{2}{*}{$\begin{array}{c}\text { HLS } \\
\text { necesarias }\end{array}$} & \multicolumn{2}{|c|}{ Diferencia } & \multirow[b]{2}{*}{$\begin{array}{l}\text { Excesos va- } \\
\text { lor absoluto }\end{array}$} & \multirow[b]{2}{*}{$\begin{array}{c}\text { Positivo } \\
\%\end{array}$} \\
\hline & & & & & & $\begin{array}{c}\text { Valor } \\
\text { absoluto }\end{array}$ & $\%$ & & \\
\hline Autónoma Barcelona .... & 413 & 20 & 1 & 136 & 188,5 & $-52,5$ & $-27,8$ & 一 & - \\
\hline Politécnica Barcelona ... & 9.949 & 1.051 & 28 & 7.336 & $5.394,9$ & $1.941,1$ & 35,98 & $2.302,1$ & 42,67 \\
\hline $\begin{array}{lllllll}\text { Córdoba } & \ldots & \ldots & \ldots & \ldots & \ldots\end{array}$ & 592 & 87 & 4 & 746 & 376,2 & 369,8 & 98,3 & 369,8 & 98,30 \\
\hline Politécnica Las Palmas .. & 814 & 115 & 5 & 826 & 585 & 241 & 41,19 & 241 & 41,19 \\
\hline Politécnica Madrid ... ... & 20.993 & 1.720 & 34 & 11.761 & $8.234,75$ & $3.526,25$ & 42,82 & $3.622,25$ & 43,98 \\
\hline $\begin{array}{lllllll}\text { Oviedo } & \ldots & \ldots & \ldots & \ldots & \ldots & \ldots\end{array}$ & 764 & 130 & 6 & 918 & 342 & 576 & 168,42 & 576 & 168,42 \\
\hline 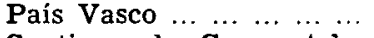 & 2.470 & 147 & 6 & 933 & 971,5 & $-38,5$ & $-3,9$ & - & - \\
\hline Santiago de Compostela. & 1.109 & 76 & $\mathbf{5}$ & 473 & 547,5 & $-74,5$ & $-13,60$ & 15,55 & 2,83 \\
\hline $\begin{array}{llllll}\text { Santander } & \ldots & \ldots & \ldots & \ldots & \ldots\end{array}$ & 358 & 70 & $\mathbf{3}$ & 530 & 237 & 293 & 123,6 & 293 & 123,60 \\
\hline $\begin{array}{lllllll}\text { Sevilla } & \ldots & \ldots & \ldots & \ldots & \ldots & \ldots\end{array}$ & 2.397 & 172 & 2 & 593 & 732 & -139 & $-18,98$ & - & - \\
\hline Politécnica Valencia .. ... & 3.464 & 382 & 14 & 2.756 & $1.807,4$ & 948,6 & 52,48 & $1.130,6$ & 62,55 \\
\hline $\begin{array}{llllll}\text { Zaragoza } & \ldots & \ldots & \ldots & \ldots & \ldots\end{array}$ & 1.029 & 65 & $\mathbf{3}$ & 494 & 411 & 83 & 20,2 & 83 & 20,20 \\
\hline $\begin{array}{llllll}\text { Valladolid } & \ldots & \ldots & \ldots & \ldots & \ldots\end{array}$ & 1.053 & 89 & 7 & 538 & 458 & 80 & 17,46 & 169 & 36,89 \\
\hline $\begin{array}{lllllll}\text { UNED } & \ldots & \ldots & \ldots & \ldots & \ldots & \ldots\end{array}$ & 900 & 15 & 2 & 246 & 267 & -21 & $-7,86$ & - & - \\
\hline Total $\ldots \ldots \ldots c c c c$ & 46.305 & 4.156 & 119 & 28.286 & $20.552,75$ & $7.733,25$ & 37,62 & $8.802,25$ & 42,82 \\
\hline
\end{tabular}




\section{CUADRO 6}

ETS

Curso 1981-82

Universidades

ETS

Autónoma Barcelona.

Politécnica Barcelona ...

Córdoba . . . . . . . . . . . ...

Politécnica Las Palmas ..

Politécnica Madrid .......

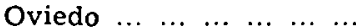

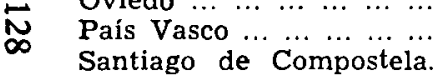

Santander

\begin{tabular}{lllllll} 
Sevilla & $\ldots$ & $\ldots$ & $\ldots$ & $\ldots$ & $\ldots$ & $\ldots$ \\
\hline
\end{tabular}

Politécnica Valencia .. ...

Zaragoza

Valladolid

UNED

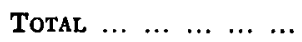

Resumen de las HLS de las ETS, según Universidades

Profesorado sin responsabilidad docente (SRD)

\begin{tabular}{|c|c|c|c|c|c|c|c|c|}
\hline \multirow[b]{2}{*}{ Alumnos } & \multirow[b]{2}{*}{$\begin{array}{l}N .^{\circ} \text { pro- } \\
\text { fesores }\end{array}$} & \multirow[b]{2}{*}{$\begin{array}{l}\text { N.o es- } \\
\text { pecialid. }\end{array}$} & \multirow[b]{2}{*}{$\begin{array}{c}\text { HLS } \\
\text { pagadas }\end{array}$} & \multirow[b]{2}{*}{$\begin{array}{c}H L S \\
\text { necesarias }\end{array}$} & \multicolumn{2}{|c|}{ Diferencia } & \multirow[b]{2}{*}{$\begin{array}{l}\text { Excesos va- } \\
\text { lor absoluto }\end{array}$} & \multirow[b]{2}{*}{$\begin{array}{c}\text { Positivo } \\
\%\end{array}$} \\
\hline & & & & & $\begin{array}{c}\text { Valor } \\
\text { absolute }\end{array}$ & $\%$ & & \\
\hline 413 & 9 & 1 & 162 & $\mathrm{~N} / \mathrm{C}$ & - & - & $\mathrm{N} / \mathrm{C}$ & $\mathrm{N} / \mathrm{C}$ \\
\hline 9.949 & 174 & 28 & 4.306 & $\mathrm{~N} / \mathrm{C}$ & - & - & $\mathrm{N} / \mathrm{C}$ & $\mathrm{N} / \mathrm{C}$ \\
\hline 592 & 60 & 4 & 1.420 & $N / C$ & - & - & $N / C$ & $\mathrm{~N} / \mathrm{C}$ \\
\hline 814 & 31 & 5 & 791 & 909 & -118 & $-12,98$ & 75 & 8,25 \\
\hline 20.993 & 311 & 33 & 9.082 & $13.030,8$ & $-3.948,8$ & $-30,30$ & $2.572,2$ & 19,73 \\
\hline 764 & 35 & 6 & 857 & 424 & 433 & 102,12 & 605 & 142,68 \\
\hline 2.470 & 30 & 6 & 818 & $1.621,5$ & $-803,5$ & $-49,5$ & - & - \\
\hline 1.109 & 9 & 5 & 144 & 955 & -811 & $-84,92$ & - & - \\
\hline 358 & 26 & 3 & 544 & 172,5 & 371,5 & 215,3 & 371,5 & 215,3 \\
\hline 2.397 & 64 & 2 & 448 & 1.034 & -586 & $-56,67$ & - & - \\
\hline 3.464 & 111 & 14 & 2.171 & 1.977 & 194 & 9,81 & 194 & 9,81 \\
\hline 1.029 & 24 & 3 & 306 & $\mathrm{~N} / \mathrm{C}$ & - & - & $\mathrm{N} / \mathrm{C}$ & $\mathrm{N} / \mathrm{C}$ \\
\hline 1.053 & 36 & 7 & 312 & 390 & -78 & -20 & - & - \\
\hline 900 & 15 & 2 & 240 & 344 & -104 & $-30,23$ & - & - \\
\hline 46.305 & 935 & 119 & 15.407 & $20.857,8$ & $-5.450,80$ & $-26,13$ & $3.817,70$ & 18,30 \\
\hline
\end{tabular}


$\mathrm{Si}$ se analiza ahora el comportamiento de los diferentes estudios que forman parte de las ETS, las conclusiones son también de gran interés (cuadro 7, segunda columna). Dos carreras están mal dotadas de profesorado: Ingeniería Química ( $-27,8$ por 100 de déficit de HLS), que sólo se estudia en un lugar, y Arquitectura que, aunque presenta un déficit de sólo un $-3,94$ por 100 de HLS, es el resultado de una desigual situación en los ocho lugares donde puede estudiarse, tanto en cantidades, como de signo.

\section{CUADRO 7}

Resumen de las HLS de las ETS, según estudios

Curso 1981-82

\begin{tabular}{|c|c|c|c|}
\hline \multirow[t]{2}{*}{ Estudios ETS } & \multirow{2}{*}{$\begin{array}{l}\text { N. de Uni- } \\
\text { versid. en } \\
\text { que se es- } \\
\text { tudia }\end{array}$} & $\begin{array}{c}\text { Profesorado } \\
\text { con responsa- } \\
\text { bilidad } \\
\text { docente }\end{array}$ & $\begin{array}{l}\text { Profesorado } \\
\text { sin responsa- } \\
\text { bilidad } \\
\text { docente }\end{array}$ \\
\hline & & $\begin{array}{l}\text { Porcentaje } \\
\text { de variación } \\
\text { de las HLS }\end{array}$ & $\begin{array}{l}\text { Porcentaje } \\
\text { de variación } \\
\text { de las HLS }\end{array}$ \\
\hline Ingeniería Química $\ldots \ldots \ldots$ & 1 & $-27,8$ & $\mathrm{~N} / \mathrm{C}$ \\
\hline $\begin{array}{llllllll}\text { Arquitectura } & \ldots & \ldots & \ldots & \ldots & \ldots & \ldots & \ldots\end{array}$ & 8 & $-3,94$ & $-80,82$ \\
\hline $\begin{array}{llllllll}\text { Aeronáuticos } & \ldots & \ldots & \ldots & \ldots & \ldots & \ldots & \ldots\end{array}$ & 1 & 61,8 & 1,5 \\
\hline $\begin{array}{llllllllll}\text { Agrónomos } & \ldots & \ldots & \ldots & \ldots & \ldots & \ldots & \ldots & \ldots\end{array}$ & 4 & 91,08 & 24,49 \\
\hline Caminos, Canales y Puertos ... ... & 4 & 63,10 & 3,49 \\
\hline $\begin{array}{lllllllll}\text { Industriales } & \ldots & \ldots & \ldots & \ldots & \ldots & \ldots & \ldots & \ldots\end{array}$ & 12 & 60,32 & $-8,40$ \\
\hline $\begin{array}{lllllllll}\text { Informática } & \ldots & \ldots & \ldots & \ldots & \ldots & \ldots & \ldots & \ldots\end{array}$ & 2 & 6,39 & $\mathrm{~N} / \mathrm{C}$ \\
\hline 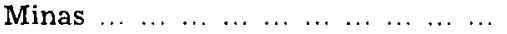 & 2 & 128,49 & 165,01 \\
\hline $\begin{array}{llllllllll}\text { Montes } & \ldots & \ldots & \ldots & \ldots & \ldots & \ldots & \ldots & \ldots & \ldots\end{array}$ & 1 & 104,4 & 32,5 \\
\hline $\begin{array}{llllllllll}\text { Navales } & \ldots & \ldots & \ldots & \ldots & \ldots & \ldots & \ldots & \ldots & \ldots\end{array}$ & 1 & 109,7 & 53,9 \\
\hline 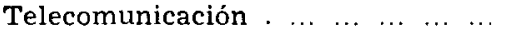 & 2 & 13,49 & 191,51 \\
\hline
\end{tabular}

Al resto de estudios les sobran HLS, aunque de forma variable: Minas (128,49 por 100 de superávit y 2 lugares posibles de estudio), Navales $(109,7$ y 1), Montes (104,4 y 1), Agrónomos (91,08 y 4), Caminos, Canales y Puertos $(63,10$ y 4$)$, Aeronáuticos $(61,8$ y 1$)$ e Industriales (60,32 y 12).

$\mathrm{Si}$ ahora pasamos al análisis del profesorado sin responsabilidad docente (SRD) y sus Horas Lectivas Semanales, hemos de hacer una advertencia previa y de importancia (cuadro 6). Aunque la situación es desigual, existen caren. cias de datos (N/C) en bastantes casos (la Autónoma de Barcelona es el más significativo), y también se tiene la sospecha de que los datos disponibles no son totalmente fiables en algunos estudios determinados. Por ello, las conclusiones que de los datos numéricos disponibles aquí se extraen, deben hacerse con cierta dosis de prevención. No obstante lo anterior, si debe huirse de extraer conclusiones muy concretas y particulares, se pueden intentar apuntar algunas de carácter global. 
Por lo que parece, existe a nivel global de Universidades un importante déficit de HLS del Profesorado Sin Responsabilidad Docente ( $-26,13$ por 100). Solamente las Universidades de Santander $(214,3$ por 100$)$, Oviedo $(102,12)$ y Politécnica de Valencia $(9,81)$ tienen sobrantes de este tipo de HLS, y el resto, o no tienen datos (N/C), o contemplan sustanciales déficits: Santiago $(-84,92$ por 100$)$, Sevilla $(-56,67)$, País Vasco $(-49,50)$, Politécnica de Madrid $(-30,30)$, UNED $(-30,23)$ y Valladolid $(-20)$.

Al centrarnos en las dos últimas columnas del citado cuadro, las que atienden solamente a los sobrantes positivos de los diferentes estudios y Universidades, la cifra varía y el signo cambia, lógicamente: un 18,30 por 100 de HLS sobran en todas las Universidades, si se eliminan los déficits y se igualan a cero, resaltando con ello aún más las desigualdades existentes entre las áreas en las que faltan las HLS y aquellas en que exceden a las necesarias. Por Universidades, se confirman las mencionadas anteriormente, añadiéndose únicamente las Politécnicas de Madrid y Las Palmas al grupo de incrementos positivos, lo que significa que en estos dos casos, y a pesar de su media negativa anterior en HLS $(-30,30$ y $-12,98$ por 100), disponen de estudios con sobrantes de estas Horas Lectivas Semanales.

En cuanto a la homogeneidad o no de las variaciones y signo de los distintos estudios que forman parte de cada Universidad, puede aventurarse una cierta tipología:

- Datos que no constan (N/C): Autónoma de Barcelona, Politécnica de Barcelona, Córdoba y Zaragoza.

- Estudios negativos: País Vasco, Santander, Sevilla, Valladolid y UNED.

- Estudios positivos: Santander.

- Estudios desiguales de signo y/o cantidad: Politécnica de Las Palmas, Politécnica de Madrid, Oviedo y Politécnica de Valencia.

Para finalizar, el análisis de estos mismos estudios de forma agrupada, y según los lugares en que se pueden cursar, da lugar a las siguientes reflexiones (véase cuadro 7 , tercera columna):

- Estudios con sobrantes de HLS del SRD: Telecomunicación (191,51 por 100), Minas (165,01), Navales (53,9), Montes (32,5), Agrónomos $(24,49)$, Caminos, Canales y Puertos $(3,49)$ y Aeronáuticos $(1,5)$.

- Estudios con déficits: Arquitectura $(-80,82$ por 100) e Industriales $(-8,40)$.

Como comentario a lo anterior, y explicando la aparente paradoja de esta mayoría de estudios con sobrantes elevados en términos relativos (con sólo un par de casos de déficits de HLS) y el elevado déficit de estas HLS visto más atrás $(-26,13$ por 100$)$, se puede decir que ello es debido al enorme peso en valores absolutos de estas dos Escuelas, sobre todo la de Arquitec- 
tura, que con -7.087 de HLS (casi -5.000 son sólo de Madrid), explican y desvirtúan el carácter relativamente equilibrado o, por lo menos, no totalmente deficitario de las Escuelas Técnicas Superiores en cuanto a las HLS del Profesorado Sin Responsabilidad Docente.

\section{Escuelas Universitarias (EE. UU.)}

Al analizar las horas lectivas semanales (HLS) del profesorado con responsabilidad docente (PRD), en el cuadro 8 , se observa que son mayoría las EE. UU. que tienen déficit; es decir, que se necesitan más HLS de las que actualmente se ofrecen. Sólo en el caso de cuatro Universidades (Barcelona Politécnica, 38,96 por 100; Córdoba, 37,72; Las Palmas Politécnica, 18,35, y Valladolid, 16,11) el balance es positivo, y aun en el caso de esta última hay que hacer notar que, aunque la suma algebraica de los porcentajes dé un resultado positivo, existen dos estudios con cantidades negativas nada despreciables. Por todo ello, el balance general de las Escuelas Universitarias en España es deficitario en cuanto al profesorado, pues frente a las $31.809,5$ HLS que serían necesarias sólo se ofrecen 27.050 , lo que supone un porcentaje de $-14,96$ por 100 .

Las Universidades peor dotadas son: Barcelona Autónoma $(-68,99$ por 100), País Vasco $(-53,22)$, Alicante $(-42,25)$, Baleares $(-41,15)$, Murcia $(-35,54)$ y La Laguna $(-33,90)$. Sin embargo, conviene analizar estas cifras con cuidado, ya que a veces no son muy representativas, pues en las EE. UU. es donde existen más lagunas en la información sobre los Planes de Estudio; así, en la mayoría de los casos éstos no están publicados y las Escuelas se rigen por las directrices generales, como es el caso de los estudios de Enfermería o Arquitectura Técnica, y, por este motivo, al menos en cinco de ellas sólo se ha tenido en cuenta el porcentaje negativo de sólo un tipo de estudio, al no poseer datos sobre los demás que se estudian en esa Universidad; el caso de Murcia es más claro, al tener información sobre dos estudios (Empresariales y Profesorado de EGB) y ser negativas las dos cantidades.

El cuadro 10 (segunda columna) nos ofrece una panorámica del estado general de los diferentes estudios que se cursan en las EE. UU. A la vista de este resumen se observa que hay casi el mismo número con cifras negativas que con positivas. Existen, sin embargo, ciertos estudios que están muy mal dotados; tal es el caso de Estudios Empresariales ( $-47,02$ por 100), Optica $(-35,16)$ o Ingeniería Técnica Topográfica $(-27,01)$, frente a otros con unas cifras de superávit bastante elevadas, y que corresponden, en su mayoría, a estudios técnicos: Ingeniería Técnica de Obras Públicas $(133,79$ por 100), Ingeniería Técnica de Minas $(31,66)$, Enfermería $(27,22)$ o Ingeniería Técnica Industrial $(24,62)$. 
CUADRO 8

Profesorado con responsabilidad docente (PRD)

\begin{tabular}{|c|c|c|c|c|c|c|c|}
\hline \multirow[t]{2}{*}{ Universidades } & \multirow[b]{2}{*}{ Alumnos } & \multirow[b]{2}{*}{$\begin{array}{l}\text { N.o pro- } \\
\text { fesores }\end{array}$} & \multirow[b]{2}{*}{$\begin{array}{l}\text { N. } .^{\circ} \text { espe- } \\
\text { cialid. }\end{array}$} & \multirow[b]{2}{*}{$\begin{array}{c}H L S \\
\text { pagadas }\end{array}$} & \multirow[b]{2}{*}{$\begin{array}{c}H L S \\
\text { necesarias }\end{array}$} & \multicolumn{2}{|c|}{ Diferenc. } \\
\hline & & & & & & $\begin{array}{l}\text { Valor } \\
\text { absoluto }\end{array}$ & $\%$ \\
\hline $\begin{array}{llllllll}\text { Alcalá } \text { de Henares } & \ldots & \ldots & \ldots & \ldots & \ldots & \ldots & \ldots\end{array}$ & 449 & 36 & $\mathrm{~N} / \mathrm{C}$ & 298 & $\mathrm{~N} / \mathrm{C}$ & $\mathrm{N} / \mathrm{C}$ & $\mathrm{N} / \mathrm{C}$ \\
\hline $\begin{array}{llllllllllll}\text { Alicante } & \ldots & \ldots & \ldots & \ldots & \ldots & \ldots & \ldots & \ldots & \ldots & \ldots & \ldots\end{array}$ & 1.119 & 77 & 4 & 177 & 306,5 & $-129,5$ & $-42,25$ \\
\hline $\begin{array}{llllllllllll}\text { Baleares } & \ldots & \ldots & \ldots & \ldots & \ldots & \ldots & \ldots & \ldots & \ldots & \ldots & \ldots\end{array}$ & 1.286 & 73 & 4 & 143 & 243 & -100 & $-41,15$ \\
\hline $\begin{array}{llllllll}\text { Barcelona Autónoma } & \ldots & \ldots & \ldots & \ldots & \ldots & \ldots\end{array}$ & 6.891 & 283 & 7 & 440 & 1.419 & -979 & $-68,99$ \\
\hline $\begin{array}{lllllllll}\text { Barcelona Central } & \ldots & \ldots & \ldots & \ldots & \ldots & \ldots & \ldots\end{array}$ & 4.879 & 294 & 4 & 239 & 280 & -41 & $-14,64$ \\
\hline $\begin{array}{llllllll}\text { Barcelona Politécnica } & \ldots & \ldots & \ldots & \ldots & \ldots & \ldots & \ldots\end{array}$ & 4.866 & 398 & 17 & 2.732 & 1.966 & 766 & 38.96 \\
\hline 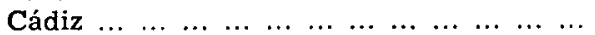 & 2.274 & 111 & 7 & 339 & 434 & -95 & $-21,89$ \\
\hline $\begin{array}{llllllllllll}\text { Córdoba } & \ldots & \ldots & \ldots & \ldots & \ldots & \ldots & \ldots & \ldots & \ldots & \ldots & \ldots\end{array}$ & 2.331 & 161 & 10 & 1.219 & 885,1 & 333,9 & 37,72 \\
\hline $\begin{array}{llllllllll}\text { Extremadura } & \ldots & \ldots & \ldots & \ldots & \ldots & \ldots & \ldots & \ldots & \ldots\end{array}$ & 1.229 & 78 & 10 & 547 & 641 & -94 & $-14,66$ \\
\hline $\begin{array}{cccccccccccc}\text { Granada } & \ldots & \ldots & \ldots & \ldots & \ldots & \ldots & \ldots & \ldots & \ldots & \ldots & \ldots\end{array}$ & 7.994 & 402 & 28 & 1.564 & 1.634 & -70 & $-4,28$ \\
\hline $\begin{array}{lllllllllll}\text { La Laguna } & \ldots & \ldots & \ldots & \ldots & \ldots & \ldots & \ldots & \ldots & \ldots & \ldots\end{array}$ & 3.760 & 146 & 4 & 311 & 470,5 & $-159,5$ & $-33,90$ \\
\hline $\begin{array}{lllllllllllll}\text { León } & \ldots & \ldots & \ldots & \ldots & \ldots & \ldots & \ldots & \ldots & \ldots & \ldots & \ldots & \ldots\end{array}$ & 1.186 & 74 & 8 & 418 & 611,5 & $-193,5$ & $-31,64$ \\
\hline $\begin{array}{llllllllll}\text { Madrid Autónoma } & \ldots & \ldots & \ldots & \ldots & \ldots & \ldots & \ldots & \ldots\end{array}$ & 3.284 & 128 & $\mathrm{~N} / \mathrm{C}$ & 1.040 & $\mathrm{~N} / \mathrm{C}$ & $\mathrm{N} / \mathrm{C}$ & $\mathrm{N} / \mathrm{C}$ \\
\hline $\begin{array}{llllllll}\text { Madrid Complutense } & \ldots & \ldots & \ldots & \ldots & \ldots & \ldots & \ldots\end{array}$ & 7.264 & 305 & 18 & 2.308 & $2.861,9$ & $-553,9$ & $-19,35$ \\
\hline $\begin{array}{llllllll}\text { Madrid Politécnica } & \ldots & \ldots & \ldots & \ldots & \ldots & \ldots & \ldots\end{array}$ & 9.565 & 542 & 25 & 3.611 & 3.963 & -352 & $-8,88$ \\
\hline $\begin{array}{llllllllllll}\text { Málaga } & \ldots & \ldots & \ldots & \ldots & \ldots & \ldots & \ldots & \ldots & \ldots & \ldots & \ldots\end{array}$ & 4.207 & 169 & 13 & 1.189 & $1.518,6$ & $-329,6$ & $-21,70$ \\
\hline $\begin{array}{llllllllllll}\text { Murcia } & \ldots & \ldots & \ldots & \ldots & \ldots & \ldots & \ldots & \ldots & \ldots & \ldots & \ldots\end{array}$ & 3.987 & 117 & 8 & 936 & 1.452 & -516 & $-35,54$ \\
\hline $\begin{array}{llllllllllll}\text { Oviedo } & \ldots & \ldots & \ldots & \ldots & \ldots & \ldots & \ldots & \ldots & \ldots & \ldots & \ldots\end{array}$ & 3.008 & 140 & 7 & 1.082 & $1.144,7$ & $-62,7$ & $-5,48$ \\
\hline $\begin{array}{lllllllllll}\text { País } & \text { Vasco } & \ldots & \ldots & \ldots & \ldots & \ldots & \ldots & \ldots & \ldots & \ldots \\
\end{array}$ & 7.484 & 279 & 6 & 391 & 836 & -445 & $-53,22$ \\
\hline 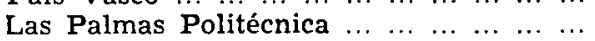 & 1.049 & 254 & 13 & 690 & 583 & 107 & 18,35 \\
\hline $\begin{array}{llllllllllll}\text { Salamanca } & \ldots & \ldots & \ldots & \ldots & \ldots & \ldots & \ldots & \ldots & \ldots & \ldots\end{array}$ & 3.377 & 152 & 9 & 461 & 607 & -146 & $-24,05$ \\
\hline $\begin{array}{llllllllllll}\text { Santander } & \ldots & \ldots & \ldots & \ldots & \ldots & \ldots & \ldots & \ldots & \ldots & \ldots\end{array}$ & 2.252 & 136 & 12 & 987 & 994 & -7 & $-0,7$ \\
\hline $\begin{array}{llllllllllll}\text { Santiago } & \ldots & \ldots & \ldots & \ldots & \ldots & \ldots & \ldots & \ldots & \ldots & \ldots & \ldots\end{array}$ & 6.867 & 346 & 18 & 769 & 978 & -209 & $-21,37$ \\
\hline $\begin{array}{llllllllllll}\text { Sevilla } & \ldots & \ldots & \ldots & \ldots & \ldots & \ldots & \ldots & \ldots & \ldots & \ldots & \ldots\end{array}$ & 6.772 & 250 & 17 & 1.558 & $1.980,8$ & $-422,8$ & $-21,34$ \\
\hline $\begin{array}{llllllll} & & & & \\
\end{array}$ & 4.127 & 226 & 1 & 305 & 357 & -52 & $-14,57$ \\
\hline $\begin{array}{llllllllllll}\text { Valencia } & \ldots & \ldots & \ldots & \ldots & \ldots & \ldots & \ldots & \ldots & \ldots & \ldots & \ldots\end{array}$ & 7.049 & 188 & 11 & 1.418 & $1.807,1$ & $-389,1$ & $-21,53$ \\
\hline $\begin{array}{llllllllllll}\text { Valladolid } & \ldots & \ldots & \ldots & \ldots & \ldots & \ldots & \ldots & \ldots & \ldots & \ldots\end{array}$ & 4.539 & 285 & 9 & 1.038 & 894 & 144 & 16,11 \\
\hline $\begin{array}{llllllllllll}\text { Zaragoza } & \ldots & \ldots & \ldots & \ldots & \ldots & \ldots & \ldots & \ldots & \ldots & \ldots\end{array}$ & 8.217 & 355 & 31 & 2.178 & $2.941,8$ & $-763,8$ & $-25,96$ \\
\hline $\begin{array}{lllllllll}\operatorname{TOTAL} & \ldots & \ldots & \ldots & \ldots & \ldots & \ldots & \ldots & \ldots\end{array}$ & 121.312 & 6.005 & & 27.050 & $31.809,5$ & $-4.759,5$ & $-14,96$ \\
\hline
\end{tabular}




\section{CUADRO 9}

EE. UU.

Curso 1981-82
Resumen de las HLS de EE. UU., según Universidades

Profesorado sin responsabilidad docente (SRD)
Universidades

EE. UU.

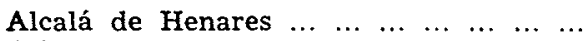

Alicante

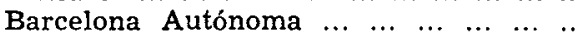

$\begin{array}{llllllll}\text { Barcelona Central } & \ldots & \ldots & \ldots & \ldots & \ldots & \ldots & \ldots\end{array}$

Barcelona Politéenica ... ... ... ... ... ... . .

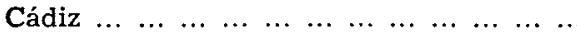

$\begin{array}{llllllllllll}\text { Córdoba } & \ldots & \ldots & \ldots & \ldots & \ldots & \ldots & \ldots & \ldots & \ldots & \ldots & \ldots\end{array}$

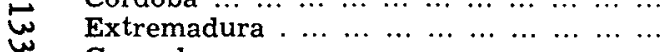

$\begin{array}{llllllllllll}\text { Granada } & \ldots & \ldots & \ldots & \ldots & \ldots & \ldots & \ldots & \ldots & \ldots & \ldots & \ldots\end{array}$

$\begin{array}{lllllllllll}\text { La Laguna } & \ldots & \ldots & \ldots & \ldots & \ldots & \ldots & \ldots & \ldots & \ldots & \ldots\end{array}$

Las Palmas Politécnica $\ldots \ldots \ldots \ldots \ldots$

$\begin{array}{llllllllllllll}\text { León } & \ldots & \ldots & \ldots & \ldots & \ldots & \ldots & \ldots & \ldots & \ldots & \ldots & \ldots & \ldots\end{array}$

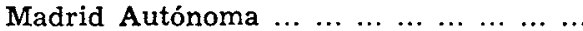

Madrid Complutense $\ldots \ldots \ldots \ldots, \ldots \ldots$

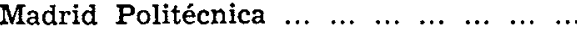

$\begin{array}{lllllllllllll}\text { Málaga } & \ldots & \ldots & \ldots & \ldots & \ldots & \ldots & \ldots & \ldots & \ldots & \ldots & \ldots\end{array}$

\begin{tabular}{lllllllllllll} 
Oviedo & $\ldots$ & $\ldots$ & $\ldots$ & $\ldots$ & $\ldots$ & $\ldots$ & $\ldots$ & $\ldots$ & $\ldots$ & $\ldots$ & $\ldots$ & $\ldots$ \\
\hline
\end{tabular}

$\begin{array}{lllllllllll}\text { País Vasco } & \ldots & \ldots & \ldots & \ldots & \ldots & \ldots & \ldots & \ldots & \ldots & \ldots\end{array}$

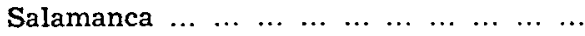

$\begin{array}{llllllllllll}\text { Santander } & \ldots & \ldots & \ldots & \ldots & \ldots & \ldots & \ldots & \ldots & \ldots & \ldots\end{array}$

$\begin{array}{lllllllllllll}\text { Santiago } & \ldots & \ldots & \ldots & \ldots & \ldots & \ldots & \ldots & \ldots & \ldots & \ldots & \ldots\end{array}$

$\begin{array}{llllllllllll}\text { Sevilla } & \ldots & \ldots & \ldots & \ldots & \ldots & \ldots & \ldots & \ldots & \ldots & \ldots & \ldots\end{array}$

Valencia

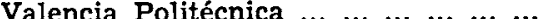

$\begin{array}{lllllllllll}\text { Valladolid } . . . & \ldots & \ldots & \ldots & \ldots & \ldots & \ldots & \ldots & \ldots & \ldots\end{array}$

Zaragoza $\ldots \ldots \ldots \ldots \ldots$

$\begin{array}{lllllllll}\operatorname{TotaL} & \ldots & \ldots & \ldots & \ldots & \ldots & \ldots & \ldots & \ldots\end{array}$

\begin{tabular}{|c|c|c|c|}
\hline Alumnos & $\begin{array}{l}\text { N. pro- } \\
\text { fesores }\end{array}$ & $\begin{array}{c}\text { N. espe- } \\
\text { cialid. }\end{array}$ & $\begin{array}{c}H L S \\
\text { pagadas }\end{array}$ \\
\hline 499 & $\mathrm{~N} / \mathrm{C}$ & $\mathrm{N} / \mathrm{C}$ & $\mathrm{N} / \mathrm{C}$ \\
\hline 1.119 & $\mathrm{~N} / \mathrm{C}$ & 4 & $\mathrm{~N} / \mathrm{C}$ \\
\hline 1.286 & 2 & 4 & 12 \\
\hline 3.866 & $N / C$ & $\mathrm{~N} / \mathrm{C}$ & $\mathrm{N} / \mathrm{C}$ \\
\hline 6.292 & 4 & 8 & 248 \\
\hline 4.866 & 57 & 17 & 1.483 \\
\hline 2.274 & 3 & 7 & 100 \\
\hline 2.331 & 11 & 7 & 355 \\
\hline 3.579 & 19 & 9 & 640 \\
\hline 7.994 & 42 & 30 & 1.340 \\
\hline 3.760 & 2 & 4 & 12 \\
\hline 1.049 & 26 & 13 & 855 \\
\hline 2.217 & 2 & 12 & 55 \\
\hline 3.284 & $\mathrm{~N} / \mathrm{C}$ & $\mathrm{N} / \mathrm{C}$ & $\mathrm{N} / \mathrm{C}$ \\
\hline 7.264 & $\mathrm{~N} / \mathrm{C}$ & 18 & $\mathrm{~N} / \mathrm{C}$ \\
\hline 9.565 & 54 & 25 & 1.540 \\
\hline 4.204 & 14 & 13 & 445 \\
\hline 2.463 & 69 & 13 & 614 \\
\hline 7.484 & $\mathrm{~N} / \mathrm{C}$ & 6 & $\mathrm{~N} / \mathrm{C}$ \\
\hline 3.377 & 19 & 13 & 620 \\
\hline 2.252 & 20 & 12 & 615 \\
\hline 6.867 & 19 & 3 & 100 \\
\hline 6.953 & 37 & 13 & 321 \\
\hline 7.049 & $N / C$ & 4 & $\mathrm{~N} / \mathrm{C}$ \\
\hline 4.127 & 40 & 1 & - \\
\hline 4.539 & 38 & 9 & 946 \\
\hline 8.217 & 36 & 4 & 200 \\
\hline 117.783 & 514 & - & 10.501 \\
\hline
\end{tabular}

Diferenc.

\begin{tabular}{c}
$\begin{array}{c}H L S \\
\text { necesarias }\end{array}$ \\
\hline
\end{tabular}

\begin{tabular}{ccc}
\hline $\begin{array}{c}\text { Valor } \\
\text { absoluto }\end{array}$ & & $\%$ \\
\cline { 1 - 1 }- & & - \\
-79 & & $-\overline{86,81}$ \\
- & & - \\
-584 & & $-70,19$ \\
-414 & & $-21,82$ \\
-110 & & $-52,38$ \\
70 & & 24,56 \\
183 & & 40,04 \\
254 & & 23,38 \\
-104 & & $-89,65$ \\
224,6 & & 35,62 \\
-35 & & $-38,88$ \\
- & - \\
-2.162 & & $-58,40$ \\
-132 & & $-22,87$ \\
$-324,2$ & & $-34,56$ \\
- & & - \\
111 & & 21,80 \\
255 & & 70,83 \\
-230 & $-69,69$ \\
-874 & $-73,13$ \\
- & - \\
-152 & $-13,84$ \\
-100 & $-33,33$ \\
\hline$-4.202,6$ & $-28,58$ \\
\hline & &
\end{tabular}


El caso de las HLS del profesorado sin responsabilidad docente (SRD) es similar al que sí la tiene (cuadro 9), pero las cifras negativas son algo más altas y es mucho mayor la cantidad de estudios para los que no se poseen datos (N/C), como, por ejemplo, el profesorado de EGB, para el que no constan horas prácticas. De manera general, puede decirse que en casi todas las Universidades (excepto Córdoba y Granada) existen cantidades negativas, y sólo en Las Palmas Politécnica, Salamanca y Santander éstas no aparecen, si bien la carencia de datos en estos centros es evidente y no permite, por tanto, extraer conclusiones definitivas.

\section{CUADRO 10}

\section{Curso 1981-82}

Resumen de las HLS de EE. UU., según estudios

\begin{tabular}{|c|c|c|c|}
\hline \multirow{2}{*}{ Estudios EE. UU. } & N. de Uni- & $P R D$ & $S R D$ \\
\hline & $\begin{array}{l}\text { des en que } \\
\text { se estudia } \\
y \text { de las } \\
\text { que se po- } \\
\text { seen datos }\end{array}$ & $\begin{array}{l}\text { Porcentaje } \\
\text { de variación } \\
\text { de las HLS }\end{array}$ & $\begin{array}{l}\text { Porcentaje } \\
\text { de variación } \\
\text { de las HLS }\end{array}$ \\
\hline Profesorado EGB & 24 & -17 & $\mathrm{~N} / \mathrm{C}$ \\
\hline Estudios Empresariales ... . . . . . . . & 20 & $-47,02$ & $-84,83$ \\
\hline $\begin{array}{lllllllll}\text { Enfermería } & \ldots & \ldots & \ldots & \ldots & \ldots & \ldots & \ldots & \ldots\end{array}$ & 10 & 27,22 & $-44,13$ \\
\hline Arquitectura Técnica ... & 8 & $-10,92$ & $-83,01$ \\
\hline Ingenieria Técnica Industrial & 14 & 24,62 & 12,87 \\
\hline Ingeniería Técnica Obras Públicas. & 4 & 133,79 & 124,97 \\
\hline Ingeniería Técnica Agrícola $\ldots \ldots \ldots$ & 7 & 10,72 & $-75,37$ \\
\hline Politécnica $\ldots \ldots \ldots \ldots \ldots \ldots$ & 2 & 1,9 & 44,69 \\
\hline Ingeneiría Técnica Aeronáutica ... & 1 & 17,98 & $-51,38$ \\
\hline Optica $\ldots \ldots \ldots \ldots \ldots \ldots$ & 1 & $-35,16$ & $\mathrm{~N} / \mathrm{C}$ \\
\hline Ingeniería Técnica Minas $\ldots \ldots \ldots$ & 6 & 31,66 & $-31,11$ \\
\hline Ingeniería Técnica Telecomunic. ... & 1 & $-22,18$ & 55,27 \\
\hline Ingeniería Técnica Topográfica . ... & 1 & $-27,01$ & $-19,59$ \\
\hline Ingeniería Técnica Naval ... ... ... & 1 & $\mathrm{~N} / \mathrm{C}$ & $\mathrm{N} / \mathrm{C}$ \\
\hline
\end{tabular}

Las cifras de las HLS de este tipo de profesorado son las siguientes: 10.501 horas ofertadas frente a $14.703,6$ necesarias, lo que da un porcentaje negativo del 28,5 por 100 , casi el doble que el que resulta del examen de las HLS para el Profesorado con Responsabilidad Docente. Las Universidades en peor situación son: La Laguna $(-89,65$ por 100$)$, Baleares $(-86,81)$, Sevilla $(-73,13)$, Barcelona Central $(-70,19)$, Santiago $(-69,69)$ y Madrid Politécnica $(-58,40)$. Como en el caso de las HLS del PRD, conviene tener en cuenta que en varias de estas Universidades sólo se poseen datos sobre uno o dos estudios cuyas cantidades son negativas, no habiendo positivas para contrarrestar; sólo en el caso de Madrid Politécnica aparece Ingeniería Técnica de Telecomunicación con un 55,27 por 100 positivo. 
Del examen de las EE. UU. por estudios (cuadro 10, tercera columna), se llega a la conclusión de que la peor situada es Empresariales $(-84,83$ por 100), seguida de Arquitectura Técnica con un porcentaje muy similar $(-83,01)$, Ingeniería Técnica Agrícola $(-75,37)$, Ingeniería Técnica Aeronáutica $(-51,38)$ y Enfermería $(-44,13)$.

Este mismo cuadro permite un análisis comparativo del conjunto de las HLS para el PRD y el SRD, observándose que hay tres estudios cuya situación es pésima para ambos tipos de horas lectivas, y son Empresariales, Arquitectura Técnica e Ingeniería Técnica Topográfica:

- Estudios Empresariales: de tener -47,02 por 100 en PRD pasa al $-84,83$ por 100 en SRD.

- Arquitectura Técnica: está en peor situación, porque frente al -10,92 por 100 pasa a un $-83,01$ por 100 , lo que supone un aumento considerable en su déficit global.

- Ingeniería Técnica Topográfica: disminuye su déficit de 27,01 por 100 en PRD pasa a 19,59 por 100 en SRD.

Las EE. UU. que están en buena situación en ambos tipos de HLS son:

- Ingeniería Técnica Industrial: 24,62 por 100 para el PRD y 12,87 por 100 para el SRD.

- Politécnica: 1,9 y 44,69 por 100 , respectivamente.

- Ingeniería Técnica de Obras Públicas: su situación es óptima, con unos porcentajes positivos bastante elevados, 133,79 por 100 en PRD y 124,97 por 100 en SRD.

\section{Conclusiones}

En primer lugar, hay que advertir que si el tamaño decidido para los grupos visto más arriba puede parecer, en cierto sentido, una importante limitación a la hora de extraer conclusiones rigurosas basadas en un posible índice numérico de la realidad de la carga docente del profesorado (toda vez que es discutible el baremo-base del grupo alumnos/profesor), no lo es tanto si cambiamos la óptica de estos valores absolutos por la de los relativos.

En efecto, en este trabajo no se trata tanto de saber exactamente cuánto déficit o superávit de HLS tiene tal o cual Facultad o ETS de cierta Universidad (pues será, por lo menos, discutible su cuantía) como la de presentar un panorama de toda la Universidad española en que comparativamente se vean las situaciones existentes según los diferentes estudios y lugares. Para este enfoque, el tamaño del grupo es irrelevante, pues aunque se acepte la modificación de éste, influirá por igual en todos los casos, de forma que los valores absolutos variarán, pero las diferencias de grado entre unos y otros se mantendrán relativamente, y las distancias entre ellos. En este sentido, cual- 
quier política de readscripción del profesorado debería aprovechar esta información, utilizándola para orientar los flujos de profesorado desde aquellos/as estudios/Universidades con claro superávit de Horas Lectivas Semanales hacia las carreras y lugares con deficiencias de profesorado.

Aunque la tentación de extraer conclusiones lo más generales y espectaculares posibles es grande, dadas las limitaciones puestas de relieve al comienzo de este trabajo, conviene contentarse con dar algunas pautas de análisis de la información elaborada y, en algún caso, apuntar posibles líneas inter. pretativas provisionales.

Téngase en cuenta que hay cuatro grupos de variables que dificultan la homogeneidad de los resultados: tipo de centro (Facultad, Escuela Técnica Superior o Escuela Universitaria), Universidad (cualquier distrito), Profesorado (Con o Sin Responsabilidad Docente, PRD o SRD, respectivamente) y estudios (cualquier carrera susceptible de ser impartida). Por eso no es de extrañar que no aparezcan fácilmente las conclusiones globales, y sí aquellas que hacen referencia a alguna de estas variables en interconexión mutua.

Casi como primera idea a retener está la de que en la Universidad española «hay de todo»: hay estudios que están bien dotados y otros que están mal, Universidades con sobrantes de profesorado en relación a sus necesidades y otras en las que escasea, etc. Si hubiera que sacar una nota común a esta situación, sería la de desigualdad entre estudios, tipos de centros, Universidades y clases de profesorado. La Universidad española, a la vista de los datos presentes, no puede decirse que esté ni muy bien ni muy mal dotada de profesorado; lo que sí puede decirse es que el que hay está muy desigualmente repartido (conviene volver a recordar que estas referencias se hacen sobre el curso 1981-82). Si partimos de la base de que estos recursos son, de cualquier forma, escasos, la desigualdad implica que los casos sobrantes agravan y hacen menos defendibles y más injustos los déficits de profesorado. Una mejor distribución del profesorado a escala nacional contribuiría, pues (sin necesidad de aumento de plantilla) a una mayor justicia distributiva interuniversitaria $y$, por ende, a una mejor calidad de la docencia; en este sentido, el desarrollo normativo que la Ley de Reforma Universitaria prevé en este punto es de esperar contribuya a racionalizar alguno de estos aspectos.

Veamos ahora algunas ideas-resumen de los diferentes tipos de centros. En cuanto a las Facultades (véase cuadro 11), puede decirse que existe un déficit de HLS del PRD del $-11,69$ por 100 a nivel nacional, mientras que en el SRD hay un superávit del $+4,36$ por 100 . Por Universidades, parece detectarse una favorable situación de las HLS del PRD en las pequeñas y/o nuevas, mientras que en el Profesorado Sin Responsabilidad Docente, las mejores son también las pequeñas y las medias, predominando las jóvenes en general; las que se encuentran en peor situación en relación al PRD son difíciles de definir, pues hay una mezcla de varias clases, si bien las de tamaño grande $y / o$ antiguas predominan entre ellas, de igual forma que en el 
CUADRO 11

Resumen general carga docente del profesorado

\begin{tabular}{|c|c|c|c|c|c|c|c|c|c|}
\hline \multirow[b]{2}{*}{$\begin{array}{l}\text { Clases } \\
\text { de } \\
\text { Centros }\end{array}$} & \multirow[b]{2}{*}{$\begin{array}{l}\text { Clase de } \\
\text { profeso- } \\
\text { rado }\end{array}$} & \multirow[b]{2}{*}{$\begin{array}{c}\text { HLS } \\
\text { pagadas }\end{array}$} & \multirow[b]{2}{*}{$\begin{array}{l}H L S \\
\text { necesa- } \\
\text { rias }\end{array}$} & \multicolumn{2}{|c|}{ Diferencia } & \multicolumn{2}{|c|}{ Universidades } & \multicolumn{2}{|c|}{ Estudios } \\
\hline & & & & $\begin{array}{l}\text { Valores } \\
\text { absolu- } \\
\text { tos }\end{array}$ & $\begin{array}{l}\text { Porcen- } \\
\text { tajes }\end{array}$ & Dos mejores & Dos peores & Dos mejores & Dos peores \\
\hline \multirow{2}{*}{$\begin{array}{l}\text { Facul- } \\
\text { tades }\end{array}$} & PRD & 97.839 & 110.785 & 12.946 & $-11,69$ & $\begin{array}{l}\text { Alcalá de Hena- } \\
\text { res }(+59,15) \\
\text { Santiago }(+23,18)\end{array}$ & $\begin{array}{l}\text { Valencia }(-38,95) \\
\text { Zaragoza }(-36,99)\end{array}$ & $\begin{array}{c}\text { Políticas y Socg. } \\
(+108,37) \\
\text { Geológicas } \\
(+38,68)\end{array}$ & $\begin{array}{l}\text { Farmacia } \\
\quad(-41,69) \\
\text { Económ. y Emp. } \\
(-37,67)\end{array}$ \\
\hline & SRD & 61.562 & 58.987 & 2.575 & $+4,36$ & $\begin{array}{l}\text { Santander } \\
\quad(+310,52) \\
\text { Málaga }(+136,69)\end{array}$ & $\begin{array}{l}\text { Valencia }(-80,00) \\
\text { Santiago }(-52,85)\end{array}$ & $\begin{array}{c}\text { Geografía e His- } \\
\text { toria }(+537,09) \\
\text { Físicas }(+149,75)\end{array}$ & $\begin{array}{l}\text { Económ. y Emp. } \\
(-44,05) \\
\text { Derecho }(-29,52)\end{array}$ \\
\hline \multirow{2}{*}{ ETS } & PRD & 28.286 & 20.553 & 7.733 & $+37,62$ & $\begin{array}{l}\text { Oviedo }(+168,42) \\
\text { Santander } \\
\quad(+123,60)\end{array}$ & $\begin{array}{l}\text { A. Barcelona } \\
(-27,80) \\
\text { Sevilla }(-18,98)\end{array}$ & $\begin{array}{l}\text { Minas }(+128,49) \\
\text { Navales }(109,7)\end{array}$ & $\begin{array}{l}\text { Ing. Quim. } \\
\quad(-27,8) \\
\text { Arquit. }(-3,94)\end{array}$ \\
\hline & SRD & 15.407 & 20.858 & -5.451 & $-26,13$ & $\begin{array}{l}\text { Santander } \\
\quad(+214,3) \\
\text { Oviedo }(+102,12)\end{array}$ & $\begin{array}{l}\text { Santiago }(-84,92) \\
\text { Sevilla }(-56,67)\end{array}$ & $\begin{array}{l}\text { Telecomunicación } \\
(+191,51) \\
\text { Minas }(165,01)\end{array}$ & $\begin{array}{l}\text { Arquit. }(-80,82) \\
\text { Indust. }(-8,40)\end{array}$ \\
\hline \multirow{2}{*}{ EE. UU. } & PRD & 27.050 & 31.809 & -4.759 & $-14,96$ & $\begin{array}{l}\text { Barcelona P. } \\
\quad(+38,96) \\
\text { Córdoba }(+37,72)\end{array}$ & $\begin{array}{l}\text { Barcelona A. } \\
\quad(-68,99) \\
\text { País V. }(-53,22)\end{array}$ & $\begin{array}{l}\text { I. T. Obras Públi- } \\
\text { cas }(+133,79) \\
\text { I. T. de Minas } \\
(+31,66)\end{array}$ & $\begin{array}{l}\text { Empres. }(-47,02) \\
\text { Optica }(-35,16)\end{array}$ \\
\hline & SRD & 10.501 & 14.704 & -4.203 & $-28,58$ & $\begin{array}{l}\text { Santander } \\
\quad(+70,83) \\
\text { Extremadura } \\
(+40,04)\end{array}$ & $\begin{array}{l}\text { La Laguna } \\
\quad(-89,65) \\
\text { Baleares }(-86,61)\end{array}$ & $\begin{array}{l}\text { I. T. Obras Públi- } \\
\text { cas }(+124,97) \\
\text { I. T. Telecom. } \\
(+55,27)\end{array}$ & $\begin{array}{l}\text { Empres. }(-54,53) \\
\text { I. T. Arquite. } \\
(-83,01)\end{array}$ \\
\hline \multirow{2}{*}{ Total } & PRD & 153.175 & 163.147 & -9.972 & $-6,11$ & & & & \\
\hline & SRD & 87.470 & 94.549 & -7.079 & $-7,52$ & & & & \\
\hline
\end{tabular}


caso del SRD, donde las peor dotadas suelen ser las medias y grandes, y/o las de creación no reciente.

En cuanto a los diferentes estudios, existe cierta uniformidad tanto en lo que se refiere al PRD como al SRD. En efecto, aunque hay casos de estudios que se salen de la tónica normal, todos los estudios de Ciencias (salvo Biológicas), Políticas, Psicología, Geografía e Historia y Filosofía y Letras se encuentran con unas HLS muy holgadas, que hacen superar las pagadas a las necesarias; en el otro lado, con déficits de cierta entidad en el PRD y en el SRD, tenemos los casos de Biológicas, Farmacia, Medicina, Económicas y Derecho.

Finalmente, y como datos un poco anecdóticos, recogemos ciertos casos sorprendentes de superávits, sobre todo por ser indicativos de esa tremenda desigualdad que se da entre unos y otros:

Ciencias, Baleares

Filos. y C. Educación, Tarragona (Barce-

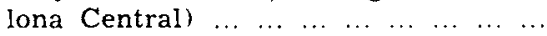

Matemáticas, La Laguna

Químicas, La Laguna..

Medicina, Madrid Complutense

Bellas Artes, Madrid Complutense

Politic. y Sociolog., Madrid Complutense.

Bellas Artes, Valencia Politécnica

Farmacia, Alcalá de Henares

Geológicas, Barcelona Central

Geograf. e Historia, Barcelona Central

Ciencias, Córdoba

Filosofía y Letras, Granada

Filosofía y Letras, Madrid Autónoma ...

Fisicas, Madrid Complutense

Económicas y Empres., Málaga

Geológicas, Oviedo

Químicas, Oviedo

Geografía e Historia, Oviedo

Químicas, País Vasco

Ciencias, Santander

Filosofia y Letras, Santander

Físicas, Sevilla

Matemáticas, Sevilla

Geografía e Historia, Sevilla

Físicas, Valencia

$\begin{array}{lcccll}\text { Sobrante de } & 147,70 & \% & \text { HLS, PRD } \\ \text { Sobrante de } & 158,82 & \% & \text { HLS, PRD } \\ \text { Sobrante de } & 261,01 & \% & \text { HLS, PRD } \\ \text { Sobrante de } & 291,60 & \% & \text { HLS, PRD } \\ \text { Sobrante de } & 254,22 & \% & \text { HLS, PRD } \\ \text { Sobrante de } & 139,67 & \% & \text { HLS, PRD } \\ \text { Sobrante de } & 108,37 & \% & \text { HLS, PRD } \\ \text { Sobrante de } & 131,67 & \% & \text { HLS, PRD } \\ \text { Sobrante de } & 133,34 & \% & \text { HLS, } & \text { SRD } \\ \text { Sobrante de } & 135,42 & \% & \text { HLS, } & \text { SRD } \\ \text { Sobrante de } & 1794,54 ! & \% & \text { HLS, } & \text { SRD } \\ \text { Sobrante de } & 130,60 & \% & \text { HLS, SRD } \\ \text { Sobrante de } & 1543,84 ! & \% & \text { HLS, } & \text { SRD } \\ \text { Sobrante de } & 186,95 & \% & \text { HLS, SRD } \\ \text { Sobrante de } & 107,89 & \% & \text { HLS, SRD } \\ \text { Sobrante de } & 226,09 & \% & \text { HLS, SRD } \\ \text { Sobrante de } & 183,63 & \% & \text { HLS, SRD } \\ \text { Sobrante de } & 138,16 & \% & \text { HLS, } & \text { SRD } \\ \text { Sobrante de } & 285,71 & \% & \text { HLS, } & \text { SRD } \\ \text { Sobrante de } & 165,71 & \% & \text { HLS, SRD } \\ \text { Sobrante de } & 548,00 & \% & \text { HLS, SRD } \\ \text { Subrante de } & 125,00 & \% & \text { HLS, SRD } \\ \text { Sobrante de } & 302,00 & \% & \text { HLS, SRD } \\ \text { Sobrante de } & 14.100,00 ! & \% & \text { HLS, SRD } \\ \text { Sobrante de } & 560,00 & \% & \text { HLS, SRD } \\ \text { Sobrante de } & 549,31 & \% & \text { HLS, SRD }\end{array}$

El otro gran grupo de centros, el de las Escuelas Técnicas Superiores (ETS), presenta una situación a nivel nacional bastante más favorable que la ya descrita para las Facultades, pues existe un sobrante de HLS para el PRD del 37,62 por 100 , que en valor absoluto son 7.733 HLS (véase cuadro 11); el déficit existente en el SRD, aunque importante (debido a lo incompleto de los Planes de Estudio), debe acogerse con cierta prevención, por lo que creemos no modifica sustancialmente el carácter globalmente positivo de las ETS, y más si se relaciona con la situación de las Facultades. 
Por Universidades, la situación no es fácil de deducir con carácter general, salvo Santander, Oviedo y Valencia Politécnica, en las que los superávits son generalizados, tanto en PRD como en SRD. Los tamaños de ellas no parecen, en este sentido, muy representativos de la existencia de déficits o superávits de las HLS.

En lo que se refiere a los estudios, sin embargo, y salvo los casos de ingenieros químicos y arquitectos (deficitarios ambos de sus HLS, tanto en el PRD como en el SRD), el resto de las carreras presenta un panorama positivo en términos generales; entre ellas destacan, por su enorme sobrante de Horas Lectivas en las dos clases de profesorado, los casos de Agrónomos, Minas, Montes y Navales, y en una sola clase del profesorado, los estudios de Aeronáuticos, Caminos, Canales y Puertos, Industriales y Telecomunicaciones.

Entre los casos destacables por su excepcionalidad en los superávits tendríamos los siguientes:

Industriales, Tarrasa

Industriales, Polit. Barcelona

Agrónomos, Córdoba

Montes, Polit. Madrid

Navales, Polit. Madrid

Minas, Oviedo

Caminos, Canales y Puertos, Santander .........

Agrónomos, Valencia Politéc.

Caminos, Canales y Puertos, Politéc. Valencia

Industriales, Valencia Politéc. ... $\ldots . . \ldots \ldots \ldots \ldots$

Minas, Politéc. Madrid ...

Telecomunicac. Politéc. Madrid

Minas, Oviedo

Caminos, Canales y Puertos, Santander

Caminos, Canales y Puertos, Politéc. Valencia ...
Sobrante de $137,9 \%$ HLS, PRD Sobrante de $135,1 \%$ HLS, PRD Sobrante de $98,3 \%$ HLS, PRD Sobrante de $104,4 \%$ HLS, PRD Sobrante de $109,7 \%$ HLS, PRD Sobrante de $228,1 \%$ HLS, PRD Sobrante de $123,6 \%$ HLS, PRD Sobrante de $101,5 \%$ HLS, PRD Sobrante de $156,4 \%$ HLS, PRD Sobrante de $122.7 \%$ HLS, PRD Sobrante de $109,1 \%$ HLS, SRD Sobrante de $191,5 \%$ HLS, SRD Sobrante de $258,5 \%$ HLS, SRD Sobrante de $215,3 \%$ HLS, SRD Sobrante de $326,8 \%$ HLS, SRD

Finalmente, por lo que se refiere a las Escuelas Universitarias (EE. UU.), se puede decir, en líneas generales, que su situación es bastante deficitaria en ambas categorías de profesorado, el PRD y el SRD, con lo que en términos relativos se sitúan algo por debajo de las Facultades y bastante peor que las ETS. Sus cifras son: déficit de $-14,96$ por 100 de HLS en el PRD, y de $-28,58$ por 100 en el SRD (véase cuadro 11 ).

$\mathrm{Si}$ se atiende a las diferentes Universidades, parece difícil extraer algún tipo de conclusión que las agrupe en uno u otro sentido; bien dotadas en ambos tipos de profesorado están las de Córdoba y Las Palmas, mientras que se hallan con déficits netos y sostenidos para el PRD y el SRD los distritos de Baleares, La Laguna y León.

Por estudios, lo más destacable dentro del capítulo superavitario es el caso de Ingeniería Técnica de Obras Públicas (+133,79 por 100 en PRD y $+124,97$ por 100 en $\mathrm{SRD}$ ), además de Industriales, con cifras algo más dis- 
cretas. La peor situación es la de Empresariales ( $-47,02$ por 100 en PRD y $-84,83$ por 100 en SRD).

Una matización que no conviene olvidar (al observar las cifras de los cuadros citados últimamente) se refiere al análisis necesario del déficit o superávit de cada Universidad, según su estructura y comportamiento de los diferentes estudios, ya que la cifra referida, aunque valiosa y significativa de la «media» general de dicha Universidad, esconde mucha información deducida de las carreras en ella impartidas, que debe ser puesta a la luz.

Piénsese, y eso ocurre siempre que se halla la media aritmética algebraica, que en su valor y magnitud influyen todos los de sus componentes, y que, por ejemplo, una Universidad con un moderado superávit medio de HLS puede ser la resultante de una mayoría de estudios con déficits de cierta entidad, y un solo superávit de importancia. Asimismo, una cantidad determinada en una Universidad puede ser lo mismo el resultado de similares castidades en sus diferentes estudios, que la media de muy desiguales cantidades en dichas carreras.

A pesar de lo dicho, y aun a riesgo de incurrir en los errores propios que toda simplificación supone, se podría obtener el siguiente perfil de la distribución del profesorado en los diferentes centros universitarios, así como de la calidad de su situación:

\begin{tabular}{|c|c|c|c|c|}
\hline $\begin{array}{l}\text { Situa- } \\
\text { ción }\end{array}$ & $\begin{array}{c}\text { Tipo } \\
\text { de centro }\end{array}$ & $\begin{array}{c}\text { Tamaño y edad } \\
\text { de la Universidad }\end{array}$ & Estudios & $\begin{array}{l}\text { Clase de } \\
\text { profeso- } \\
\text { rado }\end{array}$ \\
\hline Buena & ETS & $\begin{array}{l}\text { Universidades pequeñas } \\
\text { y jóvenes }\end{array}$ & $\begin{array}{l}\text { Técnicos } \\
\text { Ciencias } \\
\text { Geograf. e Historia }\end{array}$ & PRD \\
\hline Media & Facultades & $\begin{array}{l}\text { Universidades } \\
\text { Medias y grandes y/o } \\
\text { viejas }\end{array}$ & Desigual & - \\
\hline Mala & EE. UU. & Desigual & $\begin{array}{l}\text { Económ. y Empre. } \\
\text { Derecho } \\
\text { Arquitectura } \\
\text { Medicina } \\
\text { Farmacia }\end{array}$ & SRD \\
\hline
\end{tabular}

Al final, pues, parece desprenderse, como deducción lógica y evidente, que no puede decirse que en la Universidad española sobre o falte profesorado (pues sólo hay un déficit global en torno al 7 por 100), sino que está muy mal distribuido, afectando a todos los tipos de centros (Facultades, ETS y EE. UU.) y Universidades. 
En otro orden de cosas, parece confirmarse la correspondencia entre la distribución, buena o mala, del profesorado y la clásica dicotomía «Ciencias/ Letras», sino que rebasa ampliamente estas dos únicas variables, para tener en cuenta otros muchos aspectos.

Asimismo, esta situación de mala distribución de recursos docentes podría corregirse a corto plazo, y sin esfuerzo económico adicional ninguno, no con unas mayores dotaciones y plazas (salvo las de carácter urgente y puntual), sino por medio de la redistribución de él a escala nacional y por estudios, con lo que se lograría una mejor adecuación de la oferta existente a las necesidades planteadas, y repercutiendo todo ello en una mejor calidad, racionalidad y justicia de la docencia universitaria.

\section{Posibles lineas de actuación}

La panorámica que acabamos de ofrecer, aunque llena de matizaciones y reparos, puede decirse que se aproxima bastante a la distribución real del profesorado en nuestros centros universitarios, la cual es indudable que no puede ni debe seguir manteniéndose, pues se llegaría a una auténtica y total disfuncionalidad de la Universidad. Es necesario introducir bastantes cambios a fin de adecuar la estructura universitaria a los modelos que fijan los países más avanzados.

En este sentido, la Ley de Reforma Universitaria puede actuar al introducir algunos cambios respecto al problema que hemos analizado. Por una parte, por ejemplo, modifica el sistema de «dedicaciones» del profesorado y las reduce a dos: dedicación normal y a tiempo parcial; la primera equivale a la antigua dedicación exclusiva, pretendiéndose que la mayoría de los profesores tengan ese horario lectivo; mientras que la dedicación «a tiempo parcial» se correspondería con la «normal», y se aplicaría fundamentalmente a los profesores contratados temporalmente (Profesores Asociados y Ayudantes), con carácter excepcional y minoritario.

Asimismo, otro aspecto que se ve modificado por la Ley de Reforma Universitaria es la diversidad de categorías de profesores existentes en la actualidad. Frente a los tres niveles clásicos del Profesorado Con Responsabilidad Docente (Catedráticos Numerarios, profesores Agragedaos y profesores Adjuntos), la Ley contempla sólo dos: Catedráticos, ya sean de Universidad o de EE. UU., integrándose en esta categoría los actuales profesores Agregados, y los profesores Titulares, que agrupan a los profesores Adjuntos.

Estas dos modificaciones, sin duda, van a contribuir a una mayor clarificación de la situación del profesorado, pero no serían bastante eficaces si no se vieran acompañadas de su redistribución, por lo que deben considerarse como un paso previo para intentar una verdadera solución del problema. En este sentido, es necesario realizar un estudio en profundidad sobre la nece- 
sidad de homogeneizar los Planes de Estudio, a fin de que a igual área de conocimiento existieran unos similares Planes de Estudio, al menos en una parte mínima. Asimismo, la reordenación de las especialidades dentro de cada tipo de estudio (simplificando y justificando ampliamente su razón de ser), así como la modificación de la actualmente desequilibrada proporción entre asignaturas optativas y obligatorias, son otras tantas tareas que, en el terreno de la ordenación universitaria, deben acometerse para lograr una mejor y más científica distribución del profesorado.

Otro tema a considerar sería el de establecer unos tamaños de grupos de alumnos «tipo» (por áreas, carreras, clase de asignatura, si es teórica o práctica, etc.) que permitieran contar con unos baremos objetivos a la hora de la distribución del profesorado y un mayor aprovechamiento de las clases impartidas, evitándose la excesiva masificación o las clases semivacías. La movilidad del profesorado es, asimismo, imprescindible para lograr una mejor distribución entre todas las Universidades, y que no se vean favorecidos determinados centros o distritos solamente por su simple ubicación geográfica o su mayor influencia dentro de la administración universitaria.

Indudablemente, la tarea es muy ardua y será lenta, pero no cabe duda de que es posible de realizar, para así conseguir una Universidad mejor. 